\title{
THE PATTERSON-SULLIVAN EMBEDDING AND MINIMAL VOLUME ENTROPY FOR OUTER SPACE
}

\author{
ILYA KAPOVICH AND TATIANA NAGNIBEDA
}

\begin{abstract}
Motivated by Bonahon's result for hyperbolic surfaces, we construct an analogue of the Patterson-Sullivan-Bowen-Margulis map from the Culler-Vogtmann outer space $C V\left(F_{k}\right)$ into the space of projectivized geodesic currents on a free group. We prove that this map is a topological embedding and thus obtain a new compactification of the outer space. We also prove that for every $k \geq 2$ the minimum of the volume entropy of the universal covers of finite connected volume-one metric graphs with fundamental group of rank $k$ and without degree-one vertices is equal to $(3 k-3) \log 2$ and that this minimum is realized by trivalent graphs with all edges of equal lengths, and only by such graphs.
\end{abstract}

\section{INTRODUCTION}

A geodesic current on a word-hyperbolic group $G$ is a positive $G$-invariant Radon measure on the space $\partial^{2} G:=\{(x, y): x, y \in \partial G, x \neq y\}$, where $\partial G$ is the hyperbolic boundary of $G$ endowed with the canonical boundary topology. The study of geodesic currents on free groups is motivated by investigating geometry and dynamics of individual automorphisms, as well as of groups of automorphisms of a free group. A similar program proved to be successful in the case of fundamental groups of hyperbolic surfaces. Bonahon's foundational work [3, 4] showed the relevance of geodesic currents to the study of the geometry of the Teichmüller space and of the dynamical properties of surface homeomorphisms. Results about geodesic currents in the hyperbolic surface case can be also found in 6, 7, 17, 36, 33. and other sources. Interesting applications of geodesic currents to the study of free group automorphisms were recently obtained in [21, 22, 23, 20].

Patterson-Sullivan measures were introduced by Patterson 31 and Sullivan 38. in the context of a Kleinian group acting on the boundary of a hyperbolic space. The notion was extended by Coornaert [9] to the case of a group $G$ acting geometrically (that is isometrically, properly discontinuously and cocompactly) on a Gromov-hyperbolic geodesic metric space (see also related works [5, 8, 18, 32, 11]). Patterson-Sullivan measures on a Gromov-hyperbolic space were further studies by Furman [14]. Patterson-Sullivan measures on the universal covers of finite simplicial graphs were considered by Lyons [26] and by Coornaert and Papadopoulos [10].

2000 Mathematics Subject Classification. Primary 20F65, Secondary 05C, 37A, 37E, 57M.

Key words and phrases. free groups, metric graphs, Patterson-Sullivan measures, geodesic currents, volume entropy.

The first author was supported by the NSF grant DMS\#0404991. Both authors acknowledge the support of the Centre de Recerca Matemàtica at Barcelona and of the Swiss National Foundation for Scientific Research. 
Let us remind here briefly the definition in the case of a non-elementary group $G$ acting geometrically on a $C A T(-1)$ space $X$ (see also definition 3.6) For $s>0$ an $s$-conformal density is a $G$-equivariant family of regular Borel measures $\left(\mu_{x}\right)_{x \in X}$ on $\partial X$ that are pairwise absolutely continuous and with the property that their mutual Radon-Nikodym derivatives satisfy

$$
\frac{d \mu_{x}}{d \mu_{y}}(\xi)=e^{-s B_{\xi}(x, y)}, \quad \text { for every } x, y \in X,
$$

where for a point $\xi \in \partial X$ and for $x, y \in X, B_{\xi}(x, y)$ is a Busemann function defined by

$$
B_{\xi}(x, y):=\lim _{z \rightarrow \xi, z \in X}[d(x, z)-d(y, z)] .
$$

It turns out that there is a unique $s>0$ called the critical exponent $h(X)$ (see definition [3.4) such that a nonzero $s$-conformal density exists (and is moreover unique up to scalar multiplication.) Such a family $\left(\mu_{x}\right)_{x \in X}$ is said to be a family of Patterson-Sullivan measures on $\partial X$. The critical exponent $h(X)$ coincides with the Hausdorff dimension of $\partial X$.

Furman [14] proved, in the more general situation of a geometric action on a Gromov-hyperbolic space, that there is a unique up to scalar multiple nonzero $G$ invariant measure $\nu$ on $\partial^{2} X:=\left\{\left(\zeta_{1}, \zeta_{2}\right) \mid \zeta_{1}, \zeta_{2} \in \partial X, \zeta_{1} \neq \zeta_{2}\right\}$ in the same measure class as $\mu_{x}^{2}$. Any of the nonzero scalar multiples of $\nu$ is called an $X$-PattersonSullivan current. Via the identification between $\partial^{2} G$ and $\partial^{2} X$, this measure $\nu$ pulls back to a canonical, up to a scalar multiple, geodesic current on $G$, any nonzero scalar multiple of which is called a G-Patterson-Sullivan current.

In the case of closed hyperbolic surfaces Patterson-Sullivan currents admit several other equivalent characterizations. Let $S$ be a closed surface with a fixed hyperbolic metric $\rho$, so that $\widehat{(S, \rho)}=\mathbb{H}^{2}$. Thus $G=\pi_{1}(S)$ acts on $\mathbb{H}^{2}$ geometrically and $\mathbb{H}^{2} / G=S$. In this situation there is a natural identification between the space of $G$-invariant measures on $\partial^{2} \mathbb{H}^{2}$ and the space of shift-invariant measures on the unit tangent bundle $\mathbb{U} S$, where the $\mathbb{R}$-shift action is given by the geodesic flow on $(S, \rho)$. As shown by Kaimanovich [19, under this identification Patterson-Sullivan currents correspond precisely to Bowen-Margulis measures (or maximal entropy measures) on $\mathbb{U} S$, that is the only shift-invariant measures on $\mathbb{U} S$ whose entropy is equal to the topological entropy of the geodesic flow on $(S, \rho)$.

For closed hyperbolic surfaces $\mathbb{H}^{2}$-Patterson-Sullivan currents coincide with $\mathrm{Li}$ ouville currents corresponding to the hyperbolic structure $\rho$. Bonahon 3 , 4] proved that the map sending a marked hyperbolic structure to the corresponding projective class of Liouville currents provides a topological embedding $L: \mathcal{T}(S) \rightarrow \mathbb{P} C u r r(G)$ of the Teichmüller space $\mathcal{T}(S)$ to the compact space $\mathbb{P} C u r r(G)$ of projectivized geodesic $G$-currents.

The Culler-Vogtmann outer space 12 is a free group analogue of the Teichmüller space. For a free group $F$ of finite rank $k \geq 2$ the outer space $C V(F)$ consists of equivalence classes of free, discrete and minimal isometric actions of $F$ on $\mathbb{R}$-trees for which the quotient metric graph has volume one. Two such actions are equivalent if there is an $F$-equivariant isometry between the two trees in question.

Let $\Gamma$ be a finite connected graph with no degree-one and degree-two vertices, and let $\alpha: F \rightarrow \pi_{1}(\Gamma, p)$ be an isomorphism. Thus $\alpha$ defines a free and discrete action of $F$ on $\tilde{\Gamma}$ with the quotient $\Gamma$. Every choice $\mathcal{L}$ of a volume-one metric graph structure on $\Gamma$ (that is, assignment of positive lengths to non-oriented edges of $\Gamma$, 
so that the sum of the lengths of all edges is equal to 1 ) turns $\widetilde{\Gamma}$ into a metric tree so that the above action of $F$ on $\widetilde{\Gamma}$ becomes an action by isometries. Hence $\mathcal{L}$ defines a point in $C V(F)$. Varying the lengths of edges of $\Gamma$ gives an open simplex $W_{\alpha}$ in $C V(F)$ of dimension $N-1$, where $N$ is the number of non-oriented edges of $\Gamma$. Thus the outer space $C V(F)$ is a union of open simplices of bounded dimension.

There is a natural map $\tau: C V(F) \rightarrow \mathbb{P} C u r r(F)$ that takes a point of $C V(F)$ represented by the action of $F$ on a tree, to the projective class of $F$-PattersonSullivan currents corresponding to this action. We call $\tau$ the Patterson-Sullivan map (see Definition 7.1 for details and for the definition of the Hausdorff dimension map.) Our main result is the following statement, which parallels the above mentioned theorem of Bonahon for hyperbolic surfaces:

Theorem A. The Patterson-Sullivan map $\tau: C V(F) \rightarrow \mathbb{P} C u r r(F)$ is a topological embedding. The Hausdorff dimension map $h: C V(F) \rightarrow \mathbb{R}$ is continuous and, moreover, the restriction of $h$ to any open simplex in $C V(F)$ is real-analytic.

Injectivity of $\tau$ follows from a general result of Furman 14 proved in the context of geometric actions on Gromov-hyperbolic spaces. The main work in the present paper is in proving the continuity of $\tau$.

In the case of a closed hyperbolic surface $S$ with $G=\pi_{1}(S)$ Bonahon proved that the Liouville map $L: \mathcal{T}(S) \rightarrow \mathbb{P} C \operatorname{urr}(G)$ extends to a homeomorphism from Thurston's compactification $\widehat{\mathcal{T}(S)}$ of $\mathcal{T}(S)$ to the closure of the image of $L$. It is well-known that $\widehat{\mathcal{T}(S)}$ coincides with the length-function compactification of $\mathcal{T}(S)$.

The map $\tau: C V(F) \rightarrow \mathbb{P} C u r r(F)$ is easily seen to be $\operatorname{Out}(F)$-equivariant and the closure of the image of this map $\overline{\tau(C V(F))}$ is compact. It is therefore natural to ask if $\tau$ extends to a continuous homeomorphism (that has to be $\operatorname{Out}(F)$-equivariant) from the length function compactification $\widehat{C V(F)}$ of $C V(F)$ to $\overline{\tau(C V(F))}$. It turns out that the answer is negative in a very strong sense. Thus Kapovich and Lustig (in preparation) recently proved that there does not exist a continuous $\operatorname{Out}(F)$ equivariant map $\partial C V(F) \rightarrow \mathbb{P} C u r r(F)$ where $\partial C V(F)=\widehat{C V(F)}-C V(F)$ is the length-function boundary of $C V(F)$. Therefore any equivariant topological embedding $C V(F) \rightarrow \mathbb{P} C u r r(F)$, such as the Patterson-Sullivan map $\tau$, results in a new compactification of $C V(F)$ that is different from the standard length-function compactification. This fact is the primary motivation for proving Theorem $\mathrm{A}$ and for obtaining as explicit a description of $\tau$ as possible in the process.

A different family of continuous $\operatorname{Out}(F)$-equivariant embeddings from $C V(F)$ to $\mathbb{P} C \operatorname{urr}(F)$ was constructed by Reiner Martin [27. Unlike the Patterson-Sullivan embedding $\tau$, Martin's embeddings are not based on a natural geometric construction and use an ad-hoc procedure, where a point of $C V(F)$ is sent to an explicitly defined infinite linear combination of "counting" currents determined by conjugacy classes of elements of $F$. His construction leads to infinite dimensional compactifications of $C V(F)$.

It is well-understood that in a fairly general negatively curved setting the Hausdorff dimension of the boundary coincides with the volume entropy. If $(M, g)$ is a closed connected Riemannian manifold, then the volume entropy of $g$ is defined as

$$
h(g):=\liminf _{R \rightarrow \infty} \frac{\log \operatorname{Vol}_{\tilde{g}}(B(x, R))}{R},
$$


where $x \in \widetilde{M}$ is a base-point and $B(x, R)$ is the ball of radius $R$ and center $x$ in $\widetilde{M}$, equipped with the pullback $\tilde{g}$ of the Riemannian metric $g$. It is well known that the liminf in the formula can be replaced by lim. This definition does not depend on the choice of $x \in \widetilde{M}$, and $h(g)>0$ if and only if the group $\pi_{1}(M)$ has exponential growth. If $g$ has strictly negative sectional curvature, then $(\widetilde{M}, \tilde{g})$ is a $C A T(-1)$ space and the Hausdorff dimension of its boundary (which is also equal to the critical exponent of $(\widetilde{M}, \tilde{g}))$ is equal to the volume entropy $h(g)$. A similar statement is true for the universal cover of a compact locally $C A T(-1)$-space $K$. In that case volume has to be interpreted as counting the number of $\pi_{1}(K)$-orbit points in the ball of radius $n$ around the basepoint in $\widetilde{K}$.

For a compact connected Riemannian manifold $M$ it is natural to ask what the infimum of $h(g)$ is when $g$ varies over metrics with $\operatorname{Vol}_{g}(M)=1$ and whether this infimum is achieved. This is known as the minimal entropy problem (see discussion in [1). A famous theorem of Besson, Courtois and Gallot [1] shows that if $M$ admits a locally symmetric volume-one metric $g_{0}$ of negative curvature, then $g_{0}$ minimizes volume entropy among all volume-one metrics (see an earlier paper of Katok 24] for the case of surfaces.)

A particular case of their theorem (see [2], Section 5) says that if $\left(M, g_{0}\right)$ and $\left(M^{\prime}, g\right)$ are homotopically equivalent negatively curved compact connected Riemannian manifolds of the same dimension $n \geq 3$, and if $\left(M, g_{0}\right)$ is locally symmetric, then $h^{n}(g) \operatorname{Vol}\left(M^{\prime}, g\right) \geq h^{n}\left(g_{0}\right) \operatorname{Vol}\left(M, g_{0}\right)$. Besson, Courtois and Gallot also show that $h(g)=h\left(g_{0}\right)$ and $\operatorname{Vol}\left(M^{\prime}, g\right)=\operatorname{Vol}\left(M, g_{0}\right)$ if and only if $\left(M^{\prime}, g\right)$ is isometric to $\left(M, g_{0}\right)$.

In the last section of our paper we prove an analogue of these statements in the outer space setting.

Theorem $\mathrm{A}$ implies that the volume entropy function $h$ (which again coincides with the Hausdorff dimension of the boundary and with the critical exponent) factors to a continuous function on the moduli space $\mathcal{M}=C V(F) / O u t(F)$

$$
\bar{h}: \mathcal{M} \rightarrow \mathbb{R}_{>0} .
$$

A point in $\mathcal{M}$ is a finite connected graph $\Gamma$ without degree-one and degree-two vertices and with $\pi_{1}(\Gamma) \cong F$, endowed with the structure $\mathcal{L}$ of a volume-one metric graph. Then $\bar{h}(\mathcal{L})$ is the volume entropy of the metric tree $\partial \widetilde{\Gamma}$, where the metric on $\widetilde{\Gamma}$ is given by the lift of $\mathcal{L}$. The analogue of a locally symmetric manifold is a regular graph (i.e., such that all vertices are of the same degree) with all edges of equal length. The volume entropy of a regular tree with all edges of the same length is easy to compute explicitly. In particular, assigning the length $1 /(3 k-3)$ to each of $(3 k-3)$ non-oriented edges in a trivalent graph with the fundamental group free of rank $k$ gives a volume-one metric graph with volume entropy of its universal cover equal to $(3 k-3) \log 2$. We prove that this is precisely the minimum of the volume entropy over all finite connected metric volume-one graphs without vertices of degree one or two and with fundamental group free of rank $k$.

Remark 1.1. Note that while the entropy function is constant on $\mathcal{T}(S)$, due to the constant curvature, it is not constant on $C V(F)$. One could however make the entropy constant by changing the standard normalization (graphs being of total volume 1 ) to the (less natural) one in which the total volume of a finite connected metric graph $\Gamma$ is equal to $1 / h(\tilde{\Gamma})$. 
Theorem B. Let $F$ be a free group of rank $k \geq 2$. Then:

(1) For the function $\bar{h}: C V(F) /$ Out $(F) \rightarrow \mathbb{R}$ we have

$$
\min \bar{h}=(3 k-3) \log 2 .
$$

This minimum is realized by any regular trivalent connected graph $\Gamma$ with $\pi_{1}(\Gamma) \cong F$, (so that $\Gamma$ has $3 k-3$ non-oriented edges), where each edge of $\Gamma$ is given length $1 /(3 k-3)$.

(2) If a point of $C V(F) / O u t(F)$ realizes the minimum of $h$ then this point is a regular trivalent graph with all edges of equal lengths.

(3) We have

$$
\sup _{\mathcal{M}} \bar{h}=\infty .
$$

As an intermediate step in proving Theorem $\mathrm{B}$ we establish that among all the volume-one metric structures on an $m$-regular graph $\Gamma$ with $m \geq 3$, the volume entropy is minimized by assigning all the edges of $\Gamma$ equal lengths. This fact is a particular case of solving the minimal volume entropy problem for an arbitrary finite connected graph which was carried in a recent work of Lim [25]. Related results have also been obtained by Rivin 34. The results of Rivin 34] and of Lim 25] can be used to provide alternative proofs of parts (1) and (2) of Theorem B Nonetheless, we choose to present our proof for completeness and because it uses rather different arguments from those of Rivin and of Lim (see Section 9.) For the benefit of our topological "outer space" audience we have also incuded an account of Patterson-Sullivan measures (see Section 3), as well as tried to give concrete and explicit proofs to our main results (Sections 7,8,9.)

The authors are grateful to Florent Balacheff, David Berg, Pierre de la Harpe, Vadim Kaimanovich, Jérôme Los, Martin Lustig, Paul Schupp and Dylan Thurston for helpful discussions. We are also grateful to Frederic Paulin and Seonhee Lim for informing us about Seonhee Lim's work and to Jean-Françis Lafont for informing us about the entropy results of Rivin.

\section{Geodesic Currents}

Convention 2.1. For the remainder of the paper let $F$ be a finitely generated free group of rank $k \geq 2$. We will denote by $\partial F$ the space of ends of $F$ with the standard ends-space topology. Thus $\partial F$ is a topological space homeomorphic to the Cantor set. We shall also think about $\partial F$ as the hyperbolic boundary of $F$, endowed with the canonical boundary topology, in the sense of the theory of word-hyperbolic groups (see, for example [15]).

We set

$$
\partial^{2} F:=\{(\zeta, \xi): \zeta, \xi \in \partial F \text { and } \zeta \neq \xi\} .
$$

Definition 2.2 (Geodesic currents). A geodesic current on $F$ is a positive, finite on compact subsets $F$-invariant Borel measure on $\partial^{2} F$. We denote the space of all geodesic currents on $F$ by $C u r r(F)$. The space $C u r r(F)$ comes equipped with the weak-* topology: for $\nu_{n}, \nu \in C \operatorname{urr}(F)$ we have $\lim _{n \rightarrow \infty} \nu_{n}=\nu$ iff for every two disjoint open sets $S, S^{\prime} \subseteq \partial F$ we have $\lim _{n \rightarrow \infty} \nu_{n}\left(S \times S^{\prime}\right)=\nu\left(S \times S^{\prime}\right)$.

We say that two nonzero geodesic currents are equivalent, denoted $\nu_{1} \sim \nu_{2}$, if there exists a positive scalar $r \in \mathbb{R}$ such that $\nu_{2}=r \nu_{1}$. We consider also the space

$$
\mathbb{P} C \operatorname{urr}(F):=\{\nu \in \operatorname{Curr}(F): \nu \neq 0\} / \sim
$$


of projectivized geodesic currents on $F$, endowed with the quotient topology. We denote the $\sim$-equivalence class of a nonzero geodesic current $\nu$ by $[\nu]$.

For a (finite or infinite) graph $\Delta$, denote by $V \Delta$ the set of all vertices of $\Delta$, and denote by $E \Delta$ the set of all oriented edges of $\Delta$ (i.e., the set of all ordered pairs $(u, v)$ where $u$ and $v$ are adjacent vertices in $\Delta$.) A path $\gamma$ in $\Delta$ is a sequence of oriented edges which connects a vertex $o(\gamma)$ (origin) with a vertex $t(\gamma)$ (terminus). A path is called reduced if it does not contain a back-tracking (a path of the form $\left(e e^{-1}\right)$ ). We denote by $\mathcal{P}(\Delta)$ the set of all finite reduced paths in $\Delta$. For a vertex $x \in V \Delta$, we denote by $\mathcal{P}_{x}(\Delta)$ the collection of all $\gamma \in \mathcal{P}(\Delta)$ that begin with $x$. For $\gamma \in \mathcal{P}(\Delta)$, we denote by $a(\gamma)$ the set of all $e \in E \Delta$ such that $e \gamma \in \mathcal{P}(\Delta)$ and we denote by $b(\gamma)$ the set of all $e \in E \Delta$ such that $\gamma e \in \mathcal{P}(\Delta)$.

Definition 2.3 (Simplicial charts). Let $\Gamma$ be a finite connected graph without degree-one vertices such that $\pi_{1}(\Gamma) \cong F$. Let $\alpha: F \rightarrow \pi_{1}(\Gamma, p)$ be an isomorphism, where $p$ is a vertex of $\Gamma$. We call such $\alpha$ a simplicial chart for $F$.

Let $\alpha: F \rightarrow \pi_{1}(\Gamma, p)$ be a simplicial chart. We consider $X:=\widetilde{\Gamma}$, a topological tree, and denote the covering map from $X$ to $\Gamma$ by $j: X \rightarrow \Gamma$. For $\gamma \in \mathcal{P}(X)$ we call the reduced path $j(\gamma)$ in $\Gamma$ the label of $\gamma$. As there is only one reduced path connecting two arbitrary vertices in a tree, we will often write $[x, y]$ for a path in $X$ with origin $x$ and terminus $y$.

Let $\partial X$ denote the space of ends of $X$ with the natural ends-space topology. Then we obtain a canonical $\alpha$-equivariant homeomorphism $\hat{\alpha}: \partial F \rightarrow \partial X$, as follows. Suppose we endow $\Gamma$ with the structure of a metric graph, that is, we assign a positive length to each edge of $\Gamma$. This turns $X$ into a metric tree and the action of $\pi_{1}(\Gamma, p)$ on $X$ becomes a discrete isometric action. Moreover, $X$ is quasi-isometric to $F$ and, if $F$ is equipped with a word metric and $x_{0}$ is a lift of $p$ to $X$, then the orbit map $\tilde{\alpha}: F \rightarrow X, f \rightarrow \alpha(f) x_{0}$, is a quasi-isometry. This quasi-isometry extends to a homeomorphism $\hat{\alpha}: \partial F \rightarrow \partial X$. A crucial feature of this construction is that $\hat{\alpha}$ does not depend on the choice of a metric structure on $\Gamma$. If $\alpha$ is fixed, we will usually suppress explicit mention of $\hat{\alpha}$ and also of the map $\alpha$ itself when talking about the action of $F$ on $X$ and on $\partial X$ arising from this situation. We also denote by $\partial^{2} X$ the set of all pairs $\left(\zeta_{1}, \zeta_{2}\right)$ such that $\zeta_{1}, \zeta_{2} \in \partial X$ and $\zeta_{1} \neq \zeta_{2}$. For $\left(\zeta_{1}, \zeta_{2}\right) \in \partial^{2} X$ we denote by $\left[\zeta_{1}, \zeta_{2}\right]$ the simplicial (non-parameterized) geodesic from $\zeta_{1}$ to $\zeta_{2}$ in $X$. Thus $\left[\zeta_{1}, \zeta_{2}\right]$ is a subgraph of $X$ isomorphic to the simplicial line, together with a choice of direction on that line. We also have the identification $\hat{\alpha}: \partial^{2} F \rightarrow \partial^{2} X$.

Definition 2.4 (Cylinder sets). For every reduced path $\gamma$ in $X$ denote

$$
C y l_{X}(\gamma):=\left\{\left(\zeta_{1}, \zeta_{2}\right) \in \partial^{2} X: \gamma \subseteq\left[\zeta_{1}, \zeta_{2}\right]\right.
$$

and the orientations on $\gamma$ and on $\left[\zeta_{1}, \zeta_{2}\right]$ agree $\}$

Also, for $x=o(\gamma) \in X$ denote

$$
C y l_{x}(\gamma):=\{\zeta \in \partial X: \gamma \text { is an initial segment of }[x, \zeta]\}
$$

The collection of all sets $C y l_{X}(\gamma)$, where $\gamma$ varies over $\mathcal{P}(X)$, gives a basis of closed-open sets for $\partial^{2} X$. For any $x \in X$, the collection of all sets $C y l_{x}(\gamma)$, where $\gamma$ varies over $\mathcal{P}_{x}(X)$, gives a basis of closed-open sets for $\partial X$. Let us denote $C y l_{\alpha}(\gamma):=\hat{\alpha}^{-1} C y l_{X}(\gamma)$, so that $C y l_{\alpha}(\gamma) \subseteq \partial^{2} F$. It is easy to see that for $\nu_{n}, \nu \in$ $\operatorname{Curr}(F) \lim _{n \rightarrow \infty} \nu_{n}=\nu$ iff $\lim _{n \rightarrow \infty} \nu_{n}\left(C y l_{\alpha}(\gamma)\right)=\nu\left(C y l_{\alpha}(\gamma)\right)$ for every $\gamma \in \mathcal{P}(X)$. 
Moreover, for $\nu, \nu^{\prime} \in \operatorname{Curr}(F)$ we have $\nu=\nu^{\prime}$ iff $\nu\left(C y l_{\alpha}(\gamma)\right)=\nu^{\prime}\left(C y l_{\alpha}(\gamma)\right)$ for every $\gamma \in \mathcal{P}(X)$.

Remark 2.5. Note that for any $f \in F$ and $\gamma \in \mathcal{P}(X)$ we have $f C y l_{\alpha}(\gamma)=$ $C y l_{\alpha}(f \gamma)$. Since geodesic currents are, by definition, $F$-invariant, for a geodesic current $\nu$ and for $\gamma \in \mathcal{P}(X)$ the value $\nu\left(C y l_{\alpha}(\gamma)\right)$ only depends on the label $j(\gamma)$ of $\gamma$.

\section{Patterson-Sullivan measures and metric Graphs}

Definition 3.1 (Metric and semi-metric graph structures). A quasi-metric structure $\mathcal{L}$ on a (finite or infinite) graph $\Gamma$ is an assignment of a length $L(e) \geq 0$ to each edge $e \in E \Gamma$ of $\Gamma$. The volume of $\mathcal{L}$ is $\operatorname{vol}(\mathcal{L}):=\frac{1}{2} \sum_{e \in E \Gamma} L(e)$. A quasi-metric structure is called a semi-metric structure if $L(e)=L\left(e^{-1}\right)$ for every $e \in E \Gamma$. A semi-metric structure is called a metric structure if $L(e)>0$ for every $e \in E \Gamma$. We say that a quasi-metric structure $\mathcal{L}$ is non-singular if there is a maximal tree $T$ in $\Gamma$ such that $L(e)>0$ for every $e \in E(\Gamma-T)$. A quasi-metric structure is positive if $L(e)>0$ for every $e \in E \Gamma$.

If $\mathcal{L}$ is a semi-metric structure on $\Gamma$, let $\Gamma^{\prime}$ be the graph obtained from $\Gamma$ by contracting to points all edges of $\Gamma$ of $\mathcal{L}$-length zero. Then $\Gamma^{\prime}$ comes equipped with a canonical metric graph structure $\mathcal{L}^{\prime}$ coming from $\mathcal{L}$. We call $\left(\Gamma^{\prime}, \mathcal{L}^{\prime}\right)$ the metric graph associated to $(\Gamma, \mathcal{L})$.

Convention 3.2. Let $\mathcal{L}$ be a nonsingular semi-metric graph structure on a finite graph $\Gamma$. Let $\left(\Gamma^{\prime}, \mathcal{L}^{\prime}\right)$ be the metric graph associated to $(\Gamma, \mathcal{L})$ and let $q: \Gamma \rightarrow \Gamma^{\prime}$ be the canonical projection map.

Let $X=\widetilde{\Gamma}$ and let $j: X \rightarrow \Gamma$ be the covering map. Then $\mathcal{L}$ lifts canonically to a semi-metric graph structure $\widetilde{\mathcal{L}}$ on $X$ defined as $\widetilde{L}(e):=L(j(e))$ for every $e \in E X$. Similarly let $X^{\prime}=\widetilde{\Gamma}^{\prime}$ and let $j^{\prime}: X^{\prime} \rightarrow \Gamma^{\prime}$ be the associated covering map. Again, $\mathcal{L}^{\prime}$ lifts to a metric graph structure $\widetilde{\mathcal{L}}^{\prime}$ on $X^{\prime}$.

It is easy to see that both $j$ and $j^{\prime}$ preserve edge-lengths and that $X^{\prime}$ is obtained from $X$ by contracting all edges of length zero in $X$ to points. Thus $\left(X^{\prime}, \widetilde{\mathcal{L}}^{\prime}\right)$ is the metric graph associated to $(X, \widetilde{\mathcal{L}})$. We denote by $\widetilde{q}: X \rightarrow X^{\prime}$ the canonical projection map.

The semi-metric structure $\widetilde{\mathcal{L}}$ defines a semi-metric $d=d_{\mathcal{L}}$ on $X$ and $\widetilde{\mathcal{L}^{\prime}}$ defines a metric $d^{\prime}=d_{\mathcal{L}}^{\prime}$ on $X^{\prime}$. Moreover, $\widetilde{q}:(X, d) \rightarrow\left(X^{\prime}, d^{\prime}\right)$ is distance-preserving. Note that for both $(X, d)$ and $\left(X^{\prime}, d^{\prime}\right)$ there are obvious notions of geodesic edge-paths. In both cases we can metrize $\partial X$ and $\partial X^{\prime}$ by setting

$$
\begin{gathered}
d_{x}(\xi, \zeta):=e^{-d(x,[\xi, \zeta])} \quad \text { where } \xi, \zeta \in \partial X \\
d_{x^{\prime}}^{\prime}\left(\xi^{\prime}, \zeta^{\prime}\right):=e^{-d^{\prime}\left(x^{\prime},\left[\xi^{\prime}, \zeta^{\prime}\right]\right)} \quad \text { where } \xi^{\prime}, \zeta^{\prime} \in \partial X^{\prime},
\end{gathered}
$$

where $x \in X, x^{\prime} \in X^{\prime}$. Note that $d_{x}$ is a metric on $\partial X$, although $\mathcal{L}$ was just a semimetric structure. Moreover, if $x^{\prime}=\widetilde{q}(x)$ then the map $\widetilde{q}:\left(\partial X, d_{x}\right) \rightarrow\left(\partial X^{\prime}, d_{x^{\prime}}^{\prime}\right)$ is a homeomorphism and an isometry.

Convention 3.3. For the remainder of this section we will fix $G, X$ and the notations below to be one of the following:

(1) We consider a finitely generated group $G$ acting geometrically on a $C A T(-1)$ space $X$. 
If $x \in X$ is a base-point, the boundary $\partial X$ is metrized as follows: for two points $\xi, \zeta \in \partial X$ put

$$
d_{x}(\xi, \zeta)=\left\{\begin{array}{l}
0, \quad \text { if } \xi=\zeta, \\
\exp (-d(x,[\xi, \zeta])), \quad \text { if } \xi \neq \zeta .
\end{array}\right.
$$

(2) We consider $G=F$ a free group of finite rank $k \geq 2$ and $\alpha: F \rightarrow \pi_{1}(\Gamma, p)$ a simplicial chart for $F$, as well as a non-singular semi-metric structure $\mathcal{L}$ defining a semi-metric $d$ on $X=\widetilde{\Gamma}$. Thus $F$ acts on $X$ via $\alpha$ by $d$-preserving transformations. In this case let $q: \Gamma \rightarrow \Gamma^{\prime}, \widetilde{q}: X \rightarrow X^{\prime}, \mathcal{L}^{\prime}, d^{\prime}$ and the metrics on $\partial X$ and $\partial X^{\prime}$ be as in Convention 3.2 Thus $q_{\#} \circ \alpha: F \rightarrow$ $\pi_{1}\left(\Gamma^{\prime}, p^{\prime}\right)$ is another simplicial chart for $F$, where $p^{\prime}=q(p)$ and the map $\widetilde{q}: X \rightarrow X^{\prime}$ is $F$-equivariant.

Recall from Introduction that, for a point $\xi \in \partial X$ and for $x, y \in X$, the Busemann function is

$$
B_{\xi}(x, y):=\lim _{X \ni z \rightarrow \xi}(d(x, z)-d(y, z)) .
$$

If $x, y \in X$ and $\xi \in \partial X$ are such that $y \in[x, \xi]$ then $B_{\xi}(x, y)=d(x, y)$.

Let us denote by $M(\partial X)$ the space of all positive regular Borel measures on $\partial X$ endowed with the weak-* topology. If $\mu \in M(\partial X)$ and $g \in G$ then the measure $g_{*} \mu \in M(\partial X)$ on $\partial X$ is defined as $\left(g_{*} \mu\right)(A)=\mu\left(g^{-1} A\right)$ for a Borel subset $A \subseteq \partial X$.

Proposition-Definition 3.4 (Critical Exponent). The Poincaré series of $X$ with respect to a base-point $x \in X$ is

$$
\Pi_{x}(s):=\sum_{g \in G} e^{-s d(x, g x)} .
$$

For every $x \in X$ there exists a unique number $h \geq 0$ such that $\Pi_{x}(s)$ converges for all $s>h$ and diverges for all $s<h$. This number $h$ does not depend on $x \in X$ and is called the critical exponent. We denote it by $h=h(X)=h(G, X)$.

Remark 3.5. Coornaert discusses this definition in 9. He shows in particular that under assumptions of Convention $3.3 \Pi_{x}(h)$ diverges for every $x \in X$, and that the critical exponent coincides with the volume entropy of $X$ defined by the right-hand side of the equality:

$$
h(X)=\lim _{R \rightarrow \infty} \frac{1}{R} \log \#\{g \in G: d(x, g x) \leq R\}
$$

Definition 3.6 (Conformal density). For $s \geq 0$, a continuous map $X \rightarrow M(\partial X)$, $x \mapsto \mu_{x}$ is called an $s$-dimensional conformal density on $\partial X$ for $G$ if:

(1) The family $\left(\mu_{x}\right)_{x}$ is $G$-equivariant, that is $\mu_{g x}=\left(g^{-1}\right)_{*} \mu_{x}$ for every $x \in$ $X, g \in G$.

(2) For every $x, y \in X$

$$
\frac{d \mu_{x}}{d \mu_{y}}(\xi)=e^{-s B_{\xi}(x, y)} .
$$

(3) We have $\mu_{x}=\mu_{y}$ if $d(x, y)=0$.

In particular, we see that for each $x, y \in X$ the measures $\mu_{x}, \mu_{y}$ are absolutely continuous with respect to each other with bounded Radon-Nikodym derivatives.

The following two statements follow from the basic results established in $9,10,8$. 
Proposition-Definition 3.7 (Patterson-Sullivan measures). The critical exponent $s=h(X)$ is the only value of $s \geq 0$ such that there exists a nonzero $s$ dimensional conformal density on $\partial X$. Moreover, up to scalar multiplication, the nonzero $h$-dimensional conformal density $\left(\mu_{x}\right)_{x}$ is unique. The measures $\left(\mu_{x}\right)_{x}$ are called Patterson-Sullivan measures on $\partial X$.

Proposition 3.8. Let $\left(\mu_{x}\right)_{x}$ be a family of Patterson-Sullivan measures on $\partial X$. Then

(1) The measures $\mu_{x}$ belong to the same measure class for all $x \in X$. Each $\mu_{x}$ has no atoms and has full support on $\partial X$.

(2) For every $x \in X$ the critical exponent $h$ is equal to the Hausdorff dimension of $\left(\partial X, d_{x}\right)$. In particular, $0<h(X)<\infty$.

(3) Let $x, y \in X$ and let $m_{y}$ be the $h$-dimensional Hausdorff measure on $\left(\partial X, d_{y}\right)$. Then $m_{y}$ and $\mu_{x}$ are absolutely continuous with respect to each other and their mutual Radon-Nikodym derivatives are bounded.

Here is another useful characterization of Patterson-Sullivan measures (see, for example [14]):

Proposition 3.9. Let $h=h(X)$ be the critical exponent, and let $\left(\mu_{x}\right)_{x}$ be a family of Patterson-Sullivan measures on $\partial X$. Then for every $x \in X$ the measure $\mu_{x}$ is, up to a scalar multiple, the weak limit as $s \rightarrow h+$, of the probability measures

$$
\frac{1}{\Pi_{x}(s)} \sum_{g \in G} e^{-s d(x, g x)} \operatorname{Dirac}(g x) .
$$

Convention 3.10. Let us now concentrate our attention on the case when the acting group is a nonabelian free group $F$ of finite rank $k \geq 2$. For the remainder of this section, we assume consequently that $F, \Gamma, \Gamma^{\prime}, X, X^{\prime}, \mathcal{L}$ are as in part (2) of Convention 3.3

Then $h(F, X)=h\left(F, X^{\prime}\right)$ since for every $g \in F$ and for every $x \in X$ with $x^{\prime}=\widetilde{q}(x) \in X^{\prime}$ we have $d(x, g x)=d^{\prime}\left(x^{\prime}, g x^{\prime}\right)$. We shall denote this critical exponent by $h(\mathcal{L})$.

Moreover, suppose $\left(\mu_{x}\right)_{x}$ is a conformal $s$-density on $\partial X$. Then for any $x, y \in X$ with $d(x, y)=0$ we have $\mu_{x}=\mu_{y}$ and hence $\left(\mu_{x}\right)_{x}$ canonically factors to a conformal $s$-density $\left(\mu_{x^{\prime}}^{\prime}\right)_{x^{\prime}}$ on $\partial X^{\prime}$. Similarly, if $\left(\mu_{x^{\prime}}^{\prime}\right)_{x^{\prime}}$ is a conformal s-density on $\partial X^{\prime}$, then it canonically pulls back to a conformal $s$-density $\left(\mu_{x}\right)_{x}=\left(\left(\tilde{q}^{-1}\right)_{*} \mu_{q(x)}^{\prime}\right)_{x}$ on $\partial X$.

Remark 3.11. Let $\gamma \in \mathcal{P}(X)$ be an edge-path from a vertex $x$ to a vertex $y$ in $X$. When restricted to $C y l_{x}(\gamma)$, condition (2) of Definition 3.6 simplifies to

$$
\mu_{x}=e^{-s d(x, y)} \mu_{y} \quad \text { on } C y l_{x}(\gamma)
$$

and, in particular,

$$
\mu_{x}\left(C y l_{x}(\gamma)\right)=e^{-s d(x, y)} \mu_{y}\left(C y l_{x}(\gamma)\right) .
$$

This shows that an $s$-conformal density $\left(\mu_{x}\right)_{x}$ is uniquely determined by the values $\mu_{x}\left(C y l_{x}(f)\right)$, where $x$ varies over the vertices of $X$ and $f$ varies over all edges of $X$ with origin $x$. Moreover, in view of $F$-equivariance of $\left(\mu_{x}\right)_{x}$, it suffices to take $x$ from a bijective lift of the vertex set of $\Gamma$ to $X$. 
Convention 3.12. Let $e$ be an oriented edge of $\Gamma$ and let $\left(\mu_{x}\right)_{x}$ be an $s$-conformal density for $X$ with $s>0$. Let $f$ be a lift of $e$ to $X$ and let $x$ be the origin of $f$. We denote

$$
w_{e}=w_{e, \mathcal{L}}:=\mu_{x}\left(C y l_{x}(f)\right) .
$$

Because of $F$-equivariance of $\left(\mu_{x}\right)_{x}$ the value $w_{e}$ does not depend on the choice of the lift $f$ of $e$.

Proposition 3.13. Let $s>0$. Let $\left(\mu_{x}\right)_{x}$ be an s-conformal density for $X$. Then, for every $e \in E \Gamma$,

(1) we have $w_{e}>0$;

(2) we have

$$
w_{e}=\exp (-s L(e)) \sum_{e^{\prime} \in b(e)} w_{e^{\prime}} .
$$

Moreover, if $\left(w_{e}\right)_{e \in E \Gamma}$ satisfy conditions (1), (2) above, then there exists a unique $s$-conformal density $\left(\mu_{x}\right)_{x}$ such that for every $e \in E \Gamma$ and for every lift $f$ of $e$ to $X$ with origin $x$ we have $w_{e}=\mu_{x}\left(C y l_{x}(f)\right)$.

Proof. Suppose $\left(\mu_{x}\right)_{x}$ is an $s$-conformal density for $X$. Let $e$ be an edge of $\Gamma$ and let $f$ be a lift of $e$ to $X$ with origin $x$. Since $C y l_{x}(f) \subseteq \partial X$ is a nonempty open set, Proposition 3.8 implies that $w_{e}=\mu_{x}\left(C y l_{x}(f)\right)>0$ so that condition (1) holds. Let $y$ be the terminal vertex of $f$. For every edge $e^{\prime} \in b(e)$ there is a unique lift $f^{\prime}$ of $e^{\prime}$ to $X$ with origin $y$. Then

$$
C y l_{x}(f)=\bigsqcup_{f^{\prime}} C y l_{x}\left(f f^{\prime}\right)
$$

and hence

$$
\mu_{x}\left(C y l_{x}(f)\right)=\sum_{f^{\prime}} \mu_{x}\left(C y l_{x}\left(f f^{\prime}\right)\right) .
$$

But $C y l_{x}\left(f f^{\prime}\right)=C y l_{y}\left(f^{\prime}\right)$ and we have

$$
\mu_{x}\left(C y l_{x}\left(f f^{\prime}\right)\right)=e^{-s d(x, y)} \mu_{y}\left(C y l_{y}\left(f^{\prime}\right)\right)=e^{-s L(e)} \mu_{y}\left(C y l_{y}\left(f^{\prime}\right)\right) .
$$

Therefore

$$
w_{e}=e^{-s L(e)} \sum_{e^{\prime} \in b(e)} w_{e^{\prime}} .
$$

If $\left(w_{e}\right)_{e \in E \Gamma}$ satisfy conditions (1) and (2), then it is not hard to check that using formulae from Remark 3.11 one can define an $s$-dimensional conformal density $\left(\mu_{x}\right)_{x \in X}$, as required. We leave the details of this verification to the reader.

We conclude this section with a short note on Hausdorff measures. It follows from the definitions that for any $x, y \in X$ the metrics $d_{x}, d_{y}$ on $\partial X$ are Lipschitzequivalent to each other and hence have the same Hausdorff dimension. Let $s>0$ and let $\mathcal{H}_{x}^{s}$ be the $s$-dimensional Hausdorff measure on $\left(\partial X, d_{x}\right)$.

Let $\gamma=[x, y]$ be a geodesic segment in $X$. Then $d_{x}=e^{-d(x, y)} d_{y} \quad$ on $C y l_{x}(\gamma) \subseteq$ $\partial X$. Therefore, by definition of Hausdorff measures,

$$
\mathcal{H}_{x}^{s}=e^{-s d(x, y)} \mathcal{H}_{y}^{s} \text { on } C y l_{x}(\gamma)
$$

Thus, for $s$ equal to the Hausdorff dimension of $\partial X$, the family $\left(\mathcal{H}_{x}^{s}\right)_{x}$ is a nonzero $s$ dimensional conformal density and provides a family of Patterson-Sullivan measures on $\partial X$. In particular, if we take a lift $f$ of every edge $e$ to $X$ with origin $x$ and 
denote $\theta_{e, s}:=\mathcal{H}_{x}^{s}(C y l(f))$ for $s \geq 0$, then the numbers $\theta_{e, s}$ satisfy the system of equations $\left(^{*}\right)$ from Proposition 3.13

$$
\theta_{e, s}=\sum_{e^{\prime} \in b(e)} \exp (-s L(e)) \theta_{e^{\prime}, s} \quad e \in E \Gamma .
$$

\section{Perron-Frobenius theory for metric trees}

Systems of equations of the type $(*)$ appearing in Proposition 3.13 arise in various contexts and can be studied by the theory of Perron-Frobenius-Ruelle. The matrix $A_{\mathcal{L}}(s)$ of such a system (see Convention 4.2 for the precise definition) is a transfer operator, and the statements of Lemma 4.3 and Corollary 4.4 below are standard facts about transfer operators (see for example the article of Guillopé [16] where dynamics on metric trees is studied in detail.) In the probabilistic setting, PerronFrobenius theory can be applied to study random walks on trees with finitely many cone types (among them universal covers of finite graphs.) In particular it allows the computation of the rate of escape of a random walk and of the spectral radius of its transition operator, see [29] 30].

Below we shall give a self-contained exposition of the basic facts from the PerronFrobenius theory that we need (see 37 for a detailed exposition). We shall adapt to our situation the approach of Edgar 13 to the study of self-similar fractals through so-called Mauldin-Williams graphs 28. In particular, the proof of Lemma 4.3 below follows closely the proof of Theorem 6.6.6 in [13].

If $A$ is a matrix with real coefficients, we will denote by $r(A)$ the spectral radius of $A$. Recall that a nonnegative matrix $A$ is called irreducible if for every position $i j$ there exists an integer $n>0$ such that $\left(A^{n}\right)_{i j}>0$. The notation $A \geq 0$ means that all entries of $A$ are nonnegative and the notation $A>0$ means that all entries of $A$ are positive. If $A$ and $B$ are matrices of the same size, we write $A \leq B$ if $B-A \geq 0$ and $A<B$ if $B-A>0$.

Proposition-Definition 4.1 (Perron-Frobenius Theorem). Let $A \geq 0$ be an irreducible nonnegative $n \times n$-matrix, $n \geq 1$. Then:

(1) The number $r(A)>0$ is an eigenvalue of $A$ of multiplicity 1 .

(2) There exists a (unique up to a scalar multiple) column vector $Y>0$ such that $A Y=r(A) Y$.

(3) If $Y=\left[\begin{array}{c}y_{1} \\ \vdots \\ y_{n}\end{array}\right] \geq 0$, a nonzero column vector, and $\lambda \in \mathbb{R}$ are such that $A Y=\lambda Y$, then $\lambda=r(A)$.

(4) Suppose that $Y \geq 0$, a nonzero column vector and $\lambda \in \mathbb{R}$ are such that $A Y \leq \lambda Y$ and such that for some coordinate $i$ we have $(A Y)_{i}<\lambda y_{i}$. Then $r(A)<\lambda$.

(5) Suppose that $Y \geq 0$, a nonzero column vector, and $\lambda \in \mathbb{R}$ are such that $A Y \geq \lambda Y$. Then $r(A) \geq \lambda$.

The number $r(A)$ is called the Perron-Frobenius eigenvalue of $A$. A column eigenvector $Y>0$ such that $A Y=r(A) Y$ is called a (right) Perron-Frobenius eigenvector of $A$. 
In this situation the transposed matrix $A^{T}$ is also irreducible and $r(A)=r\left(A^{T}\right)$, so that $A$ and $A^{T}$ have the same Perron-Frobenius eigenvalue. If $U$ is a right PerronFrobenius eigenvector of $A^{T}$, the row-vector $U^{T}$ is called a left Perron-Frobenius eigenvector of $A$.

Convention 4.2. For the remainder of this section, unless specified otherwise, let $F, \Gamma, X$ be as in part (2) of Convention 3.3. Let $n=\# E \Gamma$ be the number of oriented edges of $\Gamma$ and let us fix an ordering $e_{1}, \ldots, e_{n}$ on $E \Gamma$. Also, let $\mathcal{L}$ be a positive quasi-metric or a non-singular semi-metric structure on $\Gamma$.

Let $H(\Gamma)$ denote the reduced line graph of $\Gamma$, which is defined as follows. The vertex set of $H(\Gamma)$ is $E \Gamma$. The set of oriented edges of $H(\Gamma)$ consists of all reduced paths in $\Gamma$ containing exactly two edges. An edge $\gamma=e e^{\prime}$ of $H(\Gamma)$ has the origin $e$ and the terminus $e^{\prime}$. The inverse edge of $\gamma$ is the path $\left(e^{\prime}\right)^{-1} e^{-1}$. Let $M$ be the adjacency matrix of $H(\Gamma)$, that is $M=\left(m_{i j}\right)_{i, j=1}^{n}$, where

$$
m_{i j}:= \begin{cases}1, & \text { if } e_{i} e_{j} \in \mathcal{P}(\Gamma) \\ 0, & \text { otherwise. }\end{cases}
$$

Denote $A_{\mathcal{L}}(s):=\operatorname{Diag}\left(e^{-s L\left(e_{1}\right)}, \ldots, e^{-s L\left(e_{n}\right)}\right) M$. The system (*) from part (2) of Proposition 3.13 rewrites as the matrix equation:

$$
A_{\mathcal{L}}(s)\left[\begin{array}{c}
w_{e_{1}} \\
\vdots \\
w_{e_{n}}
\end{array}\right]=\left[\begin{array}{c}
w_{e_{1}} \\
\vdots \\
w_{e_{n}}
\end{array}\right]
$$

Let $\Phi_{\mathcal{L}}(s)$ denote the spectral radius of $A_{\mathcal{L}}(s)$.

Lemma 4.3. The following hold:

(1) The matrices $A_{\mathcal{L}}(s)$ and $A_{\mathcal{L}}(s)^{T}$ are nonnegative and irreducible for every $s \in \mathbb{R}$.

(2) The function $\Phi_{\mathcal{L}}(s)$ is continuous and strictly monotone decreasing on the interval $0 \leq s<\infty$.

(3) We have $\Phi_{\mathcal{L}}(0)>1$.

(4) If $\mathcal{L}$ is a positive quasi-metric structure then $\lim _{s \rightarrow \infty} \Phi_{\mathcal{L}}(s)=0$.

Proof. Recall that $\Gamma$ is finite, connected, has no degree-one vertices and $\pi_{1}(\Gamma)$ is a free group of rank $k \geq 2$. Therefore the graph $H(\Gamma)$ is strongly connected and hence its adjacency matrix $M$ is nonnegative irreducible and the same is true for its transpose $M^{T}$. The matrix $A_{\mathcal{L}}(s)$ is obtained from $M$ by multiplying the $i$-th row of $M$ by a positive number $e^{-s L\left(e_{i}\right)}$ for each $i=1, \ldots, n$. Hence $A_{\mathcal{L}}(s)$ and $A_{\mathcal{L}}(s)^{T}$ are nonnegative and irreducible.

The continuity of $\Phi_{\mathcal{L}}(s)$ follows from its definition.

Suppose now that $0 \leq s<s^{\prime}$. Let $Y=\left[\begin{array}{c}y_{1} \\ \vdots \\ y_{n}\end{array}\right]$ be a positive Perron-Frobenius eigenvector of $A_{\mathcal{L}}(s)$, so that $A_{\mathcal{L}}(s) Y=\Phi_{\mathcal{L}}(s) Y$. Since $L\left(e_{i}\right) \geq 0$, the functions $e^{-s L\left(e_{i}\right)}$ are monotone non-increasing for each $i$. Hence component-wise $a_{i j}(s) \leq$ $a_{i j}\left(s^{\prime}\right)$ and therefore $A_{\mathcal{L}}\left(s^{\prime}\right) Y \leq A_{\mathcal{L}}(s) Y=\Phi_{\mathcal{L}}(s) Y$. Moreover, there is some edge $e_{i}$ with $L\left(e_{i}\right)>0$ and hence $\left[A_{\mathcal{L}}\left(s^{\prime}\right) Y\right]_{i}<\left[A_{\mathcal{L}}(s) Y\right]_{i}=\Phi_{\mathcal{L}}(s) y_{i}$. Therefore $\Phi_{\mathcal{L}}\left(s^{\prime}\right)<\Phi_{\mathcal{L}}(s)$, as claimed. 
Note that $A_{\mathcal{L}}(0)=M$ and $\Phi_{\mathcal{L}}(0)$ is the Perron-Frobenius eigenvalue of $M$. The fundamental group of $H(\Gamma)$ is free of rank at least two. Hence the universal cover of $H(\Gamma)$ has exponential growth, that is, the spectral radius of $M$ is bigger than 1 .

To see that (4) holds, note that if $L(e)>0$ for every $e \in E \Gamma$ then we have $\lim _{s \rightarrow \infty} \sum_{i j} a_{i j}(s)=0$. Also,

$$
A(s)\left[\begin{array}{c}
1 \\
1 \\
\vdots \\
1
\end{array}\right] \leq \sum_{i j} a_{i j}(s)\left[\begin{array}{c}
1 \\
1 \\
\vdots \\
1
\end{array}\right] .
$$

Hence $\Phi(s) \leq \sum_{i j} a_{i j}(s)$ and so $\lim _{s \rightarrow \infty} \Phi(s)=0$.

Corollary 4.4. Let $\mathcal{L}$ be a positive quasi-metric structure or a non-singular semimetric structure on $\Gamma$. Then there exists a unique $s>0$ such that $\Phi_{\mathcal{L}}(s)=1$. If $\mathcal{L}$ is a non-singular semi-metric structure then $s=h(\mathcal{L})$.

Proof. Lemma 4.3 implies that there is at most one $s>0$ such that $\Phi_{\mathcal{L}}(s)=1$. If $\mathcal{L}$ is a non-singular semi-metric structure, the existence of Patterson-Sullivan measures (Proposition-Definition 3.7) and Proposition 3.13 guarantee that when $s=h(\mathcal{L})$, the Perron-Frobenius eigenvalue of $A_{\mathcal{L}}(s)$ is equal to 1 , that is, that $\Phi_{\mathcal{L}}(h(\mathcal{L}))=1$. If $\mathcal{L}$ is a positive quasi-metric structure then parts (2), (3) and (4) of Lemma 4.3 guarantee the existence of $s>0$ such that $\Phi_{\mathcal{L}}(s)=1$.

¿From now on, given a positive quasi-metric structure (or a non-singular semimetric structure) on $\Gamma$ we will denote by $h(\mathcal{L})$ the unique value $s>0$ such that $\Phi_{\mathcal{L}}(s)=1$, and will refer to $h(\mathcal{L})$ as the volume entropy of $\mathcal{L}$.

Remark 4.5. Note that if $\mathcal{L}$ is the metric structure which assigns the same lengths to all edges in $\Gamma$, this description of $h(\mathcal{L})$ specializes to the explicit formula known for the volume entropy of uniform simplicial trees, which comes from the consideration of the corresponding subshift of finite type (see e.g. [26].)

We will now rewrite the system $A_{\mathcal{L}}(s) Y=Y$ in the form allowing to apply the Implicit Function Theorem. This system is equivalent to the following $n$ equations:

$$
e^{-s L\left(e_{i}\right)}\left(m_{i 1} y_{1}+\ldots+m_{i n} y_{n}\right)-y_{i}=0, \quad i=1, \ldots, n .
$$

To express $y_{1}, \ldots, y_{n}, s$ as implicit functions of $L\left(e_{1}\right), \ldots, L\left(e_{n}\right)$ we need an extra normalizing equation: $y_{1}^{2}+\cdots+y_{n}^{2}=1$.

Proposition 4.6. Let $L_{1}=L\left(e_{1}\right), \ldots, L_{n}=L\left(e_{n}\right)$ be a non-singular semi-metric structure or a positive quasi-metric structure $\mathcal{L}$ on $\Gamma$. We set

$$
F_{i}\left(L_{1}, \ldots, L_{n}, y_{1}, \ldots, y_{n}, s\right):=e^{-s L_{i}}\left(m_{i 1} y_{1}+\ldots+m_{i n} y_{n}\right)-y_{i}
$$

for $i=1, \ldots, n$, and

$$
F_{n+1}\left(L_{1}, \ldots, L_{n}, y_{1}, \ldots, y_{n}, s\right):=y_{1}^{2}+\cdots+y_{n}^{2}-1 .
$$

Consider the following system of $n+1$ equations in $2 n+1$ variables:

$$
F_{i}\left(L_{1}, \ldots, L_{n}, y_{1}, \ldots, y_{n}, s\right)=0, \quad i=1, \ldots, n+1
$$


Let $J$ be the Jacobian of this system, that is the $(n+1) \times(n+1)$-matrix consisting of the partial derivatives of $F_{1}, \ldots, F_{n+1}$ with respect to $y_{1}, \ldots, y_{n}, s$ :

$$
J_{i j}= \begin{cases}\frac{\partial F_{i}}{\partial y_{j}} & 1 \leq i \leq n+1,1 \leq j \leq n \\ \frac{\partial F_{i}}{\partial s} & 1 \leq i \leq n+1, j=n+1 .\end{cases}
$$

Suppose $s>0, y_{i}>0$, for $i=1, \ldots, n$, are such that $z=\left(L_{1}, \ldots, L_{n}, y_{1}, \ldots, y_{n}, s\right)$ satisfies the system (!). Then $\left.\operatorname{det} J\right|_{z} \neq 0$.

Proof. Let us compute the matrix $J$ at $z$, using the information that $z$ satisfies (!). We will denote $a_{i j}=\left(A_{\mathcal{L}}(s)\right)_{i j}=e^{-s L_{i}} m_{i j}$.

For $i \neq j, 1 \leq i, j \leq n$ we get $\frac{\partial F_{i}}{\partial y_{j}}=e^{-s L_{i}} m_{i j}=a_{i j}$. For $i=j$ we get $\frac{\partial F_{i}}{\partial y_{i}}=e^{-s L_{i}} m_{i i}-1=a_{i i}-1$. Thus in the upper left corner of $J$ we see the $n \times n$ matrix $A_{\mathcal{L}}(s)-I_{n}$.

Let us compute $\frac{\partial F_{i}}{\partial s}$. We have

$$
\frac{\partial F_{i}}{\partial s}=-L_{i} e^{-s L_{i}}\left(m_{i 1} y_{1}+\ldots+m_{i n} y_{n}\right)=-L_{i} y_{i} \quad \text { for } i=1, \ldots, n,
$$

where the last equality holds since $F_{i}(z)=0$.

Finally, the last row of $J$ obtained by differentiating $F_{n+1}=y_{1}^{2}+\cdots+y_{n}^{2}-1$ along $y_{1}, . ., y_{n}, s$ is $\left[\begin{array}{llll}2 y_{1} & 2 y_{2} & \ldots & 2 y_{n}\end{array}\right]$.

Thus

$$
J=\left[\begin{array}{cccccc}
a_{11}-1 & a_{12} & a_{13} & \ldots & a_{1 n} & -L_{1} y_{1} \\
a_{21} & a_{22}-1 & a_{23} & \ldots & a_{2 n} & -L_{2} y_{2} \\
\vdots & \vdots & \vdots & \ldots & \vdots & \vdots \\
a_{i 1} & a_{i 2} & a_{i 3} & \ldots & a_{i n} & -L_{i} y_{i} \\
\vdots & \vdots & \vdots & \ldots & \vdots & \vdots \\
a_{n 1} & a_{n 2} & a_{n 3} & \ldots & a_{n n}-1 & -L_{n} y_{n} \\
2 y_{1} & 2 y_{2} & 2 y_{3} & \ldots & 2 y_{n} & 0
\end{array}\right]
$$

We claim that the rows of the matrix $J$ are linearly independent and hence $\operatorname{det} J \neq$ 0 . The column vector $Y=\left[\begin{array}{c}y_{1} \\ \vdots \\ y_{n}\end{array}\right]$ satisfies $\left(A_{\mathcal{L}}(s)-I_{n}\right) Y=0$. This implies that the last row of $J$ is perpendicular to the first $n$ rows.

Since $Y>0$, it therefore suffices to show that the first $n$ rows of $J$ are linearly independent.

Note that $\operatorname{det}\left(A_{\mathcal{L}}(s)-I_{n}\right)=0$. However the matrix $A_{\mathcal{L}}(s)-I_{n}$ has rank $n-1$ since 1 is the Perron-Frobenius eigenvalue of $A_{\mathcal{L}}(s)$ and hence has multiplicity one. Thus, up to a scalar, there is only one nontrivial linear relation between the rows of $A_{\mathcal{L}}(s)-I_{n}$. This relation is given by the left Perron-Frobenius eigenvector $Z=\left[z_{1}, \ldots, z_{n}\right]$ of $A_{\mathcal{L}}(s)$. Indeed $Z A_{\mathcal{L}}(s)=Z$ and $Z\left[A_{\mathcal{L}}(s)-I_{n}\right]=0$. Note that $z_{i}>0$ for all $i=1, \ldots, n$.

Suppose that the first $n$ rows of $J$ are linearly dependent and that we have a nonzero row vector $Z^{\prime}$ of length $n$ such that $Z^{\prime} J_{n}=0$ where $J_{n}$ is the $n \times(n+1)$ matrix consisting of the first $n$ rows of $J$. Then $Z^{\prime}$ is also a relation between the first $n$ rows of $A_{\mathcal{L}}(s)-I_{n}$ and hence $Z^{\prime}$ is a multiple of $Z$. Thus $Z J_{n}=0$.

However, when we multiply $Z$ by the last column of $J_{n}$ to compute the $(n+1)$-st entry in $Z J_{n}$, we get $-L_{1} y_{1} z_{1}-L_{2} y_{2} z_{2}-\cdots-L_{n} y_{n} z_{n}$. 
This number is strictly negative since $L_{i} \geq 0, y_{i}>0, z_{i}>0$ for all $i=1, \ldots, n$ and there is some $i$ such that $L_{i}>0$. This gives us a contradiction with the fact that $Z J_{n}=0$. $\bar{p}$.

For the remainder of this section we will denote an $n$-tuple $\left(p_{1}, \ldots, p_{n}\right) \in \mathbb{R}^{n}$ by

Corollary 4.7. Let $L_{1}^{(0)}=L^{(0)}\left(e_{1}\right), \ldots, L_{n}^{(0)}=L^{(0)}\left(e_{n}\right)$ be a non-singular semimetric or a positive quasi-metric structure $\mathcal{L}^{(0)}$ on $\Gamma$. Suppose $s^{(0)}>0, y_{i}^{(0)}>0$, where $i=1, \ldots, n$, are such that the point $z^{(0)}=\left(\bar{L}^{(0)}, \bar{y}^{(0)}, s^{(0)}\right) \in \mathbb{R}^{2 n+1}$ satisfies the system (!). Then there exist an open neighborhood $U$ of $\bar{L}^{(0)}$ in $\mathbb{R}^{n}$ and realanalytic functions $s=s(\bar{L}), y_{i}=y_{i}(\bar{L})$ on $U$ such that for every $\bar{L} \in U$ the point

$$
\left(\bar{L}, y_{1}(\bar{L}), \ldots, y_{n}(\bar{L}), s(\bar{L})\right) \in \mathbb{R}^{2 n+1}
$$

satisfies (!) and such that $y_{i}\left(\bar{L}^{(0)}\right)=y_{i}^{(0)}, s\left(\bar{L}^{(0)}\right)=s^{(0)}$.

Moreover, whenever $\bar{L} \in U$ defines a non-singular semi-metric structure $\mathcal{L}$ on $\Gamma$, $s(\bar{L})$ is equal to the critical exponent of $\left(\widetilde{\Gamma}, d_{\mathcal{L}}\right)$ and $\left(y_{1}(\bar{L}), \ldots, y_{n}(\bar{L})\right)$ is a scalar multiple of $\left(w_{e_{1}, \mathcal{L}}, \ldots, w_{e_{n}, \mathcal{L}}\right)$.

Proof. Proposition 4.6 implies that the Implicit Function Theorem is applicable at the point $z^{(0)}$. Thus there exists an open neighborhood $U$ of $z^{(0)}$ and real-analytic functions $s=s(\bar{L}), y_{i}=y_{i}(\bar{L})$ on $U$ such that for every $\bar{L} \in U$ the point

$$
\left(\bar{L}, y_{1}(\bar{L}), \ldots, y_{n}(\bar{L}), s(\bar{L})\right) \in \mathbb{R}^{2 n+1}
$$

satisfies (!) and such that $y_{i}\left(\bar{L}^{(0)}\right)=y_{i}^{(0)}, s\left(\bar{L}^{(0)}\right)=s^{(0)}$.

Moreover, since $y_{i}^{(0)}>0$, we can choose $U$ so that $y_{i}=y_{i}(\bar{L})>0$ on $U$. Let $\bar{L} \in U$ define a non-singular semi-metric structure $\mathcal{L}$ on $\Gamma$. By Proposition 3.13 the critical exponent $h=h(\mathcal{L})$ of $\left(\widetilde{\Gamma}, d_{\mathcal{L}}\right)$ satisfies the property that $\Phi_{\mathcal{L}}(h)=1$. Also, by construction, $\Phi_{\mathcal{L}}(s(\bar{L}))=1$. Corollary 4.4 now implies that $h=s(\bar{L})$. Moreover, Proposition 3.13 and the definition of the functions $y_{i}\left(L_{1}, \ldots, L_{n}\right)$ imply that both $\left(w_{e_{1}, \mathcal{L}}, \ldots, w_{e_{n}, \mathcal{L}}\right)$ and $\left(y_{1}(\bar{L}), \ldots, y_{n}(\bar{L})\right)$ are Perron-Frobenius eigenvectors of the matrix $A_{\mathcal{L}}(h)$. Therefore they are scalar multiples of each other, as required.

\section{Patterson-Sullivan CURREnts}

The following is essentially a corollary of Proposition 1 of Furman 14 .

Proposition-Definition 5.1 (Patterson-Sullivan current). Let $G, X$ be as in Convention 3.3. Let $\left(\mu_{x}\right)_{x \in X}$ be a family of Patterson-Sullivan measures on $\partial X$ and let $\mu=\mu_{y}$ for some $y \in X$. Then there exists a unique, up to a scalar multiple, $G$ invariant and flip-invariant nonzero locally finite measure $\nu$ on $\partial^{2} X$ in the measure class of $\mu \times \mu$.

Moreover, this measure $\nu$ is of the form

$$
d \nu(\xi, \zeta)=e^{2 h(X) f_{\mu}(\xi, \zeta)} d \mu(\xi) d \mu(\zeta),
$$

where $f_{\mu}: \partial^{2} X \rightarrow \mathbb{R}_{+}$is a symmetric Borel function which is within bounded distance from the function $d(x,[\xi, \zeta])$. Such a measure $\nu$ is called an $X$-PattersonSullivan current for the action of $G$ on $X$. Since $\nu$ is unique up to a scalar multiple, its projective class $[\nu]$ is called the projective $X$-Patterson-Sullivan current. 
For the remaining part of this section let $F, \Gamma, \mathcal{L}, X, d, \alpha$ be as in Part 2) of Convention 3.3

Definition 5.2. Recall that the choice of simplicial chart $\alpha$ defines a homeomorphism $\hat{\alpha}: \partial^{2} F \rightarrow \partial^{2} X$. Let $\nu$ be an $X$-Patterson-Sullivan current. Then its pull-back $\hat{\alpha}_{*}(\nu)$ is an $F$-invariant measure on $\partial^{2} F$ which is called an $F$-PattersonSullivan current for the pair $(\alpha, \mathcal{L})$. Its projective class $[\nu]$ is called the projective $F$-Patterson-Sullivan current for the pair $(\alpha, \mathcal{L})$.

We now proceed to give an explicit formula for the $X$-Patterson-Sullivan current associated with the action of $F$ on $X$.

Proposition 5.3. Let $z \in X$, and let $h(\mathcal{L})$ be the critical exponent of $X$. Let $\left(\mu_{x}\right)_{x}$ be a family of Patterson-Sullivan measures on $\partial X$ and let $w_{e}$ be defined as in Convention 3.12

Then the measure $\nu$ on $\partial^{2} X$ given by the formula

$$
d \nu(\xi, \zeta)=e^{2 h(\mathcal{L}) d(z,[\xi, \zeta])} d \mu_{z}(\xi) d \mu_{z}(\zeta)
$$

is an X-Patterson-Sullivan current.

Moreover, for any path $\gamma=[x, y] \in \mathcal{P}(X)$ we have

$$
\nu\left(C y l_{X}(\gamma)\right)=e^{-h(\mathcal{L}) L(\gamma)}\left(\sum_{e \in b\left(e^{\prime}\right)} w_{e}\right)\left(\sum_{e \in b\left(e^{\prime \prime}\right)} w_{e}\right)
$$

where $\left(e^{\prime}\right)^{-1} \in E \Gamma$ is the label of the first edge of $\gamma$ and $e^{\prime \prime} \in E \Gamma$ is the label of the last edge of $\gamma$.

Proof. We will first show that $(\dagger)$ defines a geodesic current on $\partial X$. That is, we claim that there exists a unique geodesic current $\nu^{\prime}$ such that for every $\gamma$ as in the statement of the proposition

$$
\nu^{\prime}\left(C y l_{X}(\gamma)\right)=e^{-h(\mathcal{L}) L(\gamma)}\left(\sum_{e \in b\left(e^{\prime}\right)} w_{e}\right)\left(\sum_{e \in b\left(e^{\prime \prime}\right)} w_{e}\right) .
$$

In view of the definition of $w_{e}$ 's the above formula is equivalent to

$$
\nu^{\prime}\left(C y l_{X}(\gamma)\right)=e^{-h(\mathcal{L}) L(\gamma)} \mu_{x}\left(C y l_{y}\left(\gamma^{-1}\right)\right) \mu_{y}\left(C y l_{x}(\gamma)\right) .
$$

The uniqueness of $\nu^{\prime}$ is obvious. Also, by construction $\nu^{\prime}$ is $F$-invariant, provided that $\nu^{\prime}$ is a measure. Thus it remains to show that the above formula does define a measure on $\partial^{2} X$. To do this we need to check (see, for example, [22]) that for every $\gamma$ as above

$$
\nu^{\prime}\left(C y l_{X}(\gamma)\right)=\sum_{f \in b(\gamma)} \nu^{\prime}\left(C y l_{X}(\gamma f)\right)
$$

and

$$
\nu^{\prime}\left(C y l_{X}(\gamma)\right)=\sum_{f \in a(\gamma)} \nu^{\prime}\left(C y l_{X}(f \gamma)\right)
$$

We will verify the first formula, as the second one is completely analogous. By ( $\ddagger$ ) applied to each of the paths $\gamma f$, where $f \in b(\gamma)$, we have

$$
\begin{gathered}
\nu^{\prime}\left(C y l_{X}(\gamma f)\right)=e^{-h(\mathcal{L}) L(\gamma f)} \mu_{x}\left(C y l_{t(f)}\left(f^{-1} \gamma^{-1}\right)\right) \mu_{t(f)}\left(C y l_{x}(\gamma f)\right)= \\
e^{-h(\mathcal{L}) L(\gamma f)} \mu_{x}\left(C y l_{y}\left(\gamma^{-1}\right)\right) \mu_{t(f)}\left(C y l_{x}(\gamma f)\right)= \\
e^{-h(\mathcal{L}) L(\gamma f)} \mu_{x}\left(C y l_{y}\left(\gamma^{-1}\right)\right) \mu_{t(f)}\left(C y l_{y}(f)\right) .
\end{gathered}
$$


Since

$$
C y l_{x}(\gamma)=\bigsqcup_{f \in b(\gamma)} C y l_{x}(\gamma f)
$$

it follows that

$$
\mu_{y}\left(C y l_{x}(\gamma)\right)=\sum_{f \in b(\gamma)} \mu_{y}\left(C y l_{x}(\gamma f)\right)
$$

Therefore

$$
\begin{gathered}
\nu^{\prime}\left(C y l_{X}(\gamma)\right)=e^{-h(\mathcal{L}) L(\gamma)} \mu_{x}\left(C y l_{y}\left(\gamma^{-1}\right)\right) \mu_{y}\left(C y l_{x}(\gamma)\right)= \\
e^{-h(\mathcal{L}) L(\gamma)} \mu_{x}\left(C y l_{y}\left(\gamma^{-1}\right)\right)\left(\sum_{f \in b(\gamma)} \mu_{y}\left(C y l_{x}(\gamma f)\right)\right)= \\
e^{-h(\mathcal{L}) L(\gamma)} \mu_{x}\left(C y l_{y}\left(\gamma^{-1}\right)\right)\left(\sum_{f \in b(\gamma)} \mu_{y}\left(C y l_{y}(f)\right)\right)=
\end{gathered}
$$

by formulae in Remark 3.11

$$
\begin{gathered}
e^{-h(\mathcal{L}) L(\gamma)} \mu_{x}\left(C y l_{y}\left(\gamma^{-1}\right)\right)\left(\sum_{f \in b(\gamma)} e^{-h(\mathcal{L}) L(f)} \mu_{t(f)}\left(C y l_{y}(f)\right)\right)= \\
\sum_{f \in b(\gamma)} e^{-h(\mathcal{L}) L(\gamma f)} \mu_{x}\left(C y l_{y}\left(\gamma^{-1}\right)\right) \mu_{t(f)}\left(C y l_{y}(f)\right)= \\
\sum_{f \in b(\gamma)} \nu^{\prime}\left(C y l_{X}(\gamma f)\right) .
\end{gathered}
$$

Thus $\nu^{\prime}$ is indeed a geodesic current. We will now show that $\nu^{\prime}=\nu$, where the measure $\nu$ on $\partial^{2} X$ is defined by (a). It suffices to show that $\nu\left(C y l_{X}(\gamma)\right)=\nu^{\prime}\left(C y l_{X}(\gamma)\right)$ for every $\gamma \in \mathcal{P}(X)$. Let $\gamma=[x, y] \in \mathcal{P}(X)$.

As $\nu$ is independent of the choice of $z$, we can suppose without loss of generality that $d(z,[x, y])>0$.

Let $z^{\prime} \in[x, y]$ be such that $d\left(z, z^{\prime}\right)=d(z,[x, y])$. Then

$$
\begin{gathered}
\nu\left(C y l_{X}(\gamma)\right)=e^{2 h d\left(z, z^{\prime}\right)} \mu_{z}\left(C y l_{z}([z, x])\right) \mu_{z}\left(C y l_{z}([z, y])\right)= \\
e^{2 h d\left(z, z^{\prime}\right)} e^{-h d\left(z, z^{\prime}\right)} \mu_{z^{\prime}}\left(C y l_{z^{\prime}}\left(\left[z^{\prime}, x\right]\right)\right) e^{-h d\left(z, z^{\prime}\right)} \mu_{z^{\prime}}\left(C y l_{z^{\prime}}\left(\left[z^{\prime}, y\right]\right)\right)= \\
\mu_{z^{\prime}}\left(C y l_{z^{\prime}}\left(\left[z^{\prime}, x\right]\right)\right) \mu_{z^{\prime}}\left(C y l_{z^{\prime}}\left(\left[z^{\prime}, y\right]\right)\right)= \\
e^{-h d\left(z^{\prime}, x\right)} \mu_{x}\left(C y l_{z^{\prime}}\left(\left[z^{\prime}, x\right]\right)\right) e^{-h d\left(z^{\prime}, y\right)} \mu_{y}\left(C y l_{z^{\prime}}\left(\left[z^{\prime}, y\right]\right)\right)= \\
e^{-h d(x, y)} \mu_{x}\left(C y l_{z^{\prime}}\left(\left[z^{\prime}, x\right]\right)\right) \mu_{y}\left(C y l_{z^{\prime}}\left(\left[z^{\prime}, y\right]\right)\right)= \\
e^{-h d(x, y)} \mu_{x}\left(C y l_{y}([y, x])\right) \mu_{y}\left(C y l_{x}([x, y])\right)= \\
e^{-h L(\gamma)} \mu_{x}\left(C y l_{y}\left(\gamma^{-1}\right)\right) \mu_{y}\left(C y l_{x}(\gamma)\right)=\nu^{\prime}\left(C y l_{X}(\gamma)\right) .
\end{gathered}
$$

Therefore $\nu=\nu^{\prime}$, which completes the proof of Proposition 5.3

\section{The Culler-Vogtmann outer space}

The Culler-Vogtmann outer space, introduced by Culler and Vogtmann in a seminal paper [12, is a free group analogue of the Teichmüller space of a closed surface of negative Euler characteristic. We refer the reader to the original paper [12] and to a survey paper [39] for a detailed discussion of the basic facts listed in this section and for the further references. 
Definition 6.1 (Outer space). Let $F$ be a free group of finite rank $k \geq 2$. A marked metric graph structure on $F$ is a pair $(\alpha, \mathcal{L})$, where $\alpha: F \rightarrow \pi_{1}(\Gamma, p)$ is a simplicial chart for $F$ and $\mathcal{L}$ is a metric structure on $\Gamma$. A marked metric graph structure is minimal if $\Gamma$ has no degree-one and degree-two vertices.

Two marked metric graph structures $\left(\alpha_{1}: F \rightarrow \pi_{1}\left(\Gamma_{1}, p_{1}\right), \mathcal{L}_{1}\right)$ and $\left(\alpha_{2}: F \rightarrow\right.$ $\left.\pi_{1}\left(\Gamma_{2}, p_{2}\right), \mathcal{L}_{2}\right)$ are equivalent if there exist an isometry $\iota:\left(\Gamma_{1}, \mathcal{L}_{1}\right) \rightarrow\left(\Gamma_{2}, \mathcal{L}_{2}\right)$ and a path $v$ from $\iota\left(p_{1}\right)$ to $p_{2}$ in $\Gamma_{2}$ such that

$$
\left(\iota_{\#} \circ \alpha_{1}\right)(f)=v \alpha_{2}(f) v^{-1}
$$

for every $f \in F$. Clearly, minimality is preserved by equivalence of marked metric graph structures.

The Culler-Vogtmann outer space $C V(F)$ consists of equivalence classes of all volume-one minimal marked metric graph structures on $F$.

Definition 6.2 (Elementary charts). Let $\alpha: F \rightarrow \pi_{1}(\Gamma, p)$ be a simplicial chart for $F$, where $\Gamma$ has no degree-one and degree-two vertices.

For each non-singular semi-metric structure $\mathcal{L}$ on $\Gamma$ let $\Gamma^{\prime}, \mathcal{L}^{\prime}$ and $q$ be as in Convention 3.2 Then $q_{\#} \circ \alpha: F \rightarrow \pi_{1}\left(\Gamma^{\prime}, q(p)\right)$ is a simplicial chart for $F$ and $\left(q_{\#} \circ \alpha, \mathcal{L}^{\prime}\right)$ is a minimal marked metric graph structure on $F$.

Denote by $S(\Gamma)$ the set of all volume-one non-singular semi-metric structures on $\Gamma$. Note that if $\Gamma$ has $N$ non-oriented edges, then $S(\Gamma)$ is embedded as a subset of $\mathbb{R}^{n}$. We topologize $S(\Gamma)$ accordingly.

It is not hard to see that for two non-singular semi-metric structures $\mathcal{L}_{1}, \mathcal{L}_{2}$ on $\Gamma$ the pairs $\left(q_{\#} \circ \alpha, \mathcal{L}_{1}^{\prime}\right)$ and $\left(q_{\#} \circ \alpha, \mathcal{L}_{2}^{\prime}\right)$ are equivalent if and only if $\mathcal{L}_{1}=\mathcal{L}_{2}$. Thus $\alpha$ defines an injective map $\lambda_{\alpha}: S(\Gamma) \rightarrow C V(F), \lambda_{\alpha}: \mathcal{L} \mapsto\left(q_{\#} \circ \alpha, \mathcal{L}^{\prime}\right)$. This map $\lambda_{\alpha}$ is called the elementary chart in $C V(F)$ corresponding to $\alpha$.

Let now $S_{+}(\Gamma)$ denote the set of all metric structures on $\Gamma$. If $\Gamma$ has $n$ oriented edges then $S_{+}(\Gamma)$ is an open simplex of dimension $n / 2-1$ in $\mathbb{R}^{n}$ and $S_{+}(\Gamma)$ is dense in $S(\Gamma)$.

Definition 6.3 (Topology on the outer space). The outer space $C V(F)$ is endowed with the weakest topology for which every elementary chart is a topological embedding.

As explained in [12], the outer space $C V(F)$ is a union of open simplices of the form $\lambda_{\alpha}\left(S_{+}(\Gamma)\right)$, where $\lambda_{\alpha}$ is as in Definition 6.2

One can also view $C V(F)$ as the space of projectivized hyperbolic length functions on $F$ corresponding to free and discrete isometric actions of $F$ on $\mathbb{R}$-trees.

Definition 6.4 (Projectivized length functions). Let $F \operatorname{Len}(F)$ denote the space of all hyperbolic length functions $\ell: F \rightarrow \mathbb{R}$ on $F$ corresponding to free and discrete isometric actions of $F$ on $\mathbb{R}$-trees. The space $F \operatorname{Len}(F)$ is endowed with the weak topology of pointwise convergence.

We will say that two length functions in $F \operatorname{Len}(F)$ are equivalent if they are scalar multiples of each other, and will denote by $\mathbb{P} F \operatorname{Len}(F)$ the space of equivalence classes of elements of $F \operatorname{Len}(F)$, endowed with the quotient topology. The equivalence class of $\ell \in F \operatorname{Len}(F)$ is denoted $[\ell]$. For each $\ell \in F \operatorname{Len}(F)$ there exists a free discrete minimal isometric action of $F$ on an $\mathbb{R}$-tree $X_{\ell}$ such that $\ell$ is the hyperbolic length function for this action. Moreover, the tree $X_{\ell}$ and the corresponding action of $F$ are unique up to an equivariant isometry. Let $\Gamma_{\ell}$ denote the metric graph $X_{\ell} / F$. 
Let $F \operatorname{Len}_{1}(F)$ denote the set of all $\ell \in F \operatorname{Len}(F)$ such that $\Gamma_{\ell}$ has volume one. Note that every equivalence class $[\ell] \in \mathbb{P} F \operatorname{Len}(F)$ has a unique representative in $F \operatorname{Len}_{1}(F)$. For each $\ell \in F \operatorname{Len}(F)$ the action of $F$ on $X_{\ell}$ defines an isomorphism $\alpha_{\ell}: F \rightarrow \pi_{1}\left(\Gamma_{\ell}, p\right)$, where $p \in V \Gamma_{\ell}$. Let $\mathcal{L}_{\ell}$ denote the metric structure on $\Gamma_{\ell}$ inherited from $X_{\ell}$. Note that the equivalence class of the marked metric graph structure $\left(\alpha_{\ell}, \mathcal{L}_{\ell}\right)$ on $F$ does not depend on the choice of $p$.

The following statement is well-known and can be derived from results of [12]. It shows that the outer space $C V(F)$ is homeomorphic to the spaces $F \operatorname{Len}_{1}(F)$ and $\mathbb{P} F \operatorname{Len}(F)$.

Proposition 6.5. (1) The restriction of the quotient map [] : FLen $(F) \rightarrow$ $\mathbb{P} F$ Len $(F)$ to $F$ Len $_{1}(F)$ is a homeomorphism whose image is $\mathbb{P} F$ Len $(F)$. Thus $F$ Len $_{1}(F)$ is canonically homeomorphic to $\mathbb{P} F$ Len $(F)$.

(2) Let $\varrho: F L e n_{1}(F) \rightarrow C V(F)$ be the map that takes each $\ell \in F \operatorname{Len}_{1}(F)$ to the equivalence class of the marked structure $\left(\alpha_{\ell}, \mathcal{L}_{\ell}\right)$ on $F$. Then $\varrho:$ $F_{\text {Len }}(F) \rightarrow C V(F)$ is a homeomorphism.

\section{Proof of the Main Result}

If $\left(\alpha_{1}: F \rightarrow \pi_{1}\left(\Gamma_{1}, p_{1}\right), \mathcal{L}_{1}\right)$ and $\left(\alpha_{2}: F \rightarrow \pi_{1}\left(\Gamma_{2}, p_{2}\right), \mathcal{L}_{2}\right)$ are two equivalent pairs representing the same point $\eta \in C V(F)$, then $\mathbb{R}$-trees $X_{1}=\widetilde{\Gamma}_{1}$ and $X_{2}=\widetilde{\Gamma}_{2}$ are $F$-equivariantly isometric and the corresponding hyperbolic length functions are equal. Hence it follows from Proposition 2 of Furman 14] (and it is also easy to see this directly) that the projective $F$-Patterson-Sullivan currents corresponding to $\left(\alpha_{1}, \mathcal{L}_{1}\right)$ and $\left(\alpha_{2}, \mathcal{L}_{2}\right)$ coincide (see Definition [5.2). Hence the following map is well-defined:

Definition 7.1 (Patterson-Sullivan map and Hausdorff dimension map). Let $F$ be a free group of finite rank $k \geq 2$ and let $C V(F)$ denote the outer space.

Let $\eta \in C V(F)$. Thus $\eta$ is represented as an equivalence class of $(\alpha, \mathcal{L})$, where $\alpha: F \rightarrow \pi_{1}(\Gamma, p)$ is a simplicial chart on $F$ such that $\Gamma$ is a finite connected graph without degree-one and degree-two vertices and where $\mathcal{L}$ is a volume-one metric structure on $\Gamma$. Consider $X=\widetilde{\Gamma}$ and let $d$ be the metric on $X$ induced by $\mathcal{L}$. Define $\tau(\eta)$ to be the projective $F$-Patterson-Sullivan current on $F$ corresponding to $(\alpha, \mathcal{L})$. Also define $h(\eta)$ to be the Hausdorff dimension of $\partial X$ (which, as we have seen, is equal to the critical exponent $h(\mathcal{L})$.)

This defines a map $\tau: C V(F) \rightarrow \mathbb{P} C u r r(F)$, which we will call the PattersonSullivan map, and a map $h: C V(F) \rightarrow \mathbb{R}$, which we will call the Hausdorff dimension map.

Theorem 7.2. The Patterson-Sullivan map $\tau: C V(F) \rightarrow \mathbb{P} C u r r(F)$ is a topological embedding. The Hausdorff dimension map $h: C V(F) \rightarrow \mathbb{R}$ is continuous and, moreover, the restriction of $h$ to any open simplex in $C V(F)$ is real-analytic.

Proof. Since $C V(F)$ is locally compact, in order to prove that $\tau$ is a topological embedding it suffices to show that $\tau$ is continuous and injective.

Recall the identification of $C V(F)$ with $\mathbb{P} F \operatorname{Len}(F)$ from Proposition 6.5] If $\tau\left(\left[\ell_{1}\right]\right)=\tau\left(\left[\ell_{2}\right]\right)$ for $\ell_{1}, \ell_{2} \in F \operatorname{Len}(F)$ then Theorem 2 of Furman [14 implies that there is $r>0$ such that $r \ell_{1}=\ell_{2}$ and hence $\left[\ell_{1}\right]=\left[\ell_{2}\right]$, and $\tau$ is injective. 
We now establish that $\tau$ and $h$ are continuous. Since every point of the outer space is contained in finitely many elementary charts, it suffices to prove that $\tau$ and $h$ are continuous on the image of every elementary chart in $C V(F)$.

Let $\alpha: F \rightarrow \pi_{1}(\Gamma, p)$ be a simplicial chart for $F$, where $\Gamma$ has no degree-one and degree-two vertices. Let $\lambda_{\alpha}$ be the elementary chart in $C V(F)$ determined by $\alpha$. Recall that the image $\operatorname{Im}\left(\lambda_{\alpha}\right)$ of $\lambda_{\alpha}$ consists of all points of $C V(F)$ corresponding to volume-one semi-metric structures on $\Gamma$ where all the edges with zero length are contained in a (possibly empty) subtree of $\Gamma$. Corollary 4.7 and formula $(\dagger)$ in Proposition 5.3 imply that $\left.\tau\right|_{\operatorname{Im}\left(\lambda_{\alpha}\right)}$ and $\left.h\right|_{\operatorname{Im}\left(\lambda_{\alpha}\right)}$ are continuous and, moreover, the restriction of $h$ to the interior of $\operatorname{Im}\left(\lambda_{\alpha}\right)$ is real-analytic.

Remark 7.3 (Out $(F)$-Equivariance). It is easy to see that the Patterson-Sullivan map $\tau$ is equivariant with respect to the left action of $O u t(F)$ and, in fact, a similar statement holds in the general word-hyperbolic context considered by Furman [14. It is even easier to see that $h$ is constant on each $O u t(F)$-orbit and thus factors to a continuous map on the moduli space $\bar{h}: C V(F) / O u t(F) \rightarrow \mathbb{R}$.

Indeed, suppose $\left(\alpha: F \rightarrow \pi_{1}(\Gamma, p), \mathcal{L}\right)$ represents a point $\eta \in C V(F)$ and let $\phi \in \operatorname{Aut}(F)$. Let $X=\widetilde{\Gamma}$, equipped with the metric $d$ induced by $\mathcal{L}$.

By definition of the left action of $A u t(F)$ (and of $O u t(F)$ ) on $C V(F)$, the point $\phi \eta \in C V(F)$ is the equivalence class of $\left(\phi^{-1} \circ \alpha, \mathcal{L}\right)$. For both $\eta$ and $\phi \eta$ the metric graph $(\Gamma, \mathcal{L})$ is the same. This already implies that $h(\eta)=h(\phi \eta)$.

The action of $F$ on $X$ corresponding to $\phi \eta$ is obtained from the $F$-action on $X$ corresponding to $\eta$ by a pre-composition with $\phi^{-1}$. The definitions imply that if $\left(\mu_{x}\right)_{x}$ is a family of Patterson-Sullivan measures on $\partial X$ corresponding to the action of $F$ on $X$ via $\alpha$, then $\left(\mu_{x}\right)_{x}$ is also a family of Patterson-Sullivan measures on $\partial X$ corresponding to the action of $F$ on $X$ via $\phi^{-1} \circ \alpha$. Hence if $\nu$ is an $X$-PattersonSullivan current corresponding to the action of $F$ on $X$ via $\alpha$, then $\nu$ is also an $X$-Patterson-Sullivan current corresponding to the action of $F$ on $X$ via $\phi^{-1} \circ \alpha$.

Denote $\nu_{1}:=\hat{\alpha}_{*}(\nu)$ and $\nu_{2}:=\left[\hat{\phi}^{-1} \circ \hat{\alpha}\right]_{*}(\nu)$, so that $\tau(\eta)=\left[\nu_{1}\right]$ and $\tau(\phi \eta)=\left[\nu_{2}\right]$. Definitions then imply that $\nu_{2}=\left(\hat{\phi}^{-1}\right)_{*} \nu_{1}$, that is, for any Borel subset $A \subseteq \partial^{2} F$ we have $\nu_{2}(S)=\nu_{1}\left(\hat{\phi}^{-1}(A)\right)$. By definition of the left action of $A u t(F)$ on $\operatorname{Curr}(F)$ (see [22]) we have $\left(\phi \nu_{1}\right)(A)=\nu_{1}\left(\hat{\phi}^{-1}(A)\right)$. Thus $\nu_{2}=\phi \nu_{1}$ and hence $\tau(\phi \eta)=\phi(\tau \eta)$, as claimed.

\section{The Minimal VOLUME ENTROPY PROBLEM}

Our goal in this section is to prove parts (1) and (3) of Theorem B from the Introduction. For the remainder of this section let $k \geq 2$, and let $\Gamma$ be a finite connected graph whose fundamental group $F=\pi_{1}(\Gamma, p)$ with respect to a base vertex $p \in V \Gamma$ is free of rank $k$. Let $X=\widetilde{(\Gamma, p)}$, and let $x_{0} \in V X$ be a fixed lift of $p$.

Let $w \in \mathcal{P}(\Gamma)$ and $e \in E \Gamma$. We denote by $\langle e, w\rangle$ the number of occurrences of $e$ in $w$. Let $\mathcal{L}$ be a positive quasi-metric structure on $\Gamma$. Let $w \in \mathcal{P}(\Gamma)$ be a reduced path. Then

$$
L_{\mathcal{L}}(w)=\sum_{e \in E \Gamma}\langle e, w\rangle L_{\mathcal{L}}(e)
$$

The key step in the proof of Theorem Bis the following statement, which provides a sharp bound for the volume entropy of (regular) $m$-valent metric graphs. Note 
that an $m$-valent graph with the fundamental group of rank $k$ has $m(k-1) /(m-2)$ non-oriented edges.

Proposition 8.1. For $m \geq 3$ suppose $\Gamma$ is a finite regular $m$-valent graph (i.e., every vertex has degree $m$ ) with fundamental group free of rank $k \geq 2$. Let $\mathcal{L}$ be a volume-one positive quasi-metric structure on $\Gamma$. Then

$$
h(\mathcal{L}) \geq \frac{m(k-1)}{m-2} \log (m-1) .
$$

The following lemma which we will use in the proof of Proposition 8.1 follows directly from the definition of the matrix $A_{\mathcal{L}}(s)$.

Lemma 8.2. Let $\mathcal{L}$ be a positive quasi-metric structure on $\Gamma$. Then for any integer $t \geq 1$ and for any position ij we have that

$$
\left[A_{\mathcal{L}}(s)^{t}\right]_{i j}=e^{s L\left(e_{j}\right)} \sum_{v} e^{-s L(v)}
$$

where the summation is taken over all reduced paths $v$ of edge-length $t$ with the first edges $e_{i}$ and the last edge $e_{j}$.

Proof of Proposition 8.1. We consider the simple non-backtracking random walk on $\Gamma$. This walk can be thought of as a finite state Markov process with the state set $E \Gamma$ and with transition probabilities, for $e, e^{\prime} \in E \Gamma$, defined as

$$
p\left(e, e^{\prime}\right)=\left\{\begin{array}{l}
\frac{1}{m-1}, \quad \text { if } e e^{\prime} \in \mathcal{P}(\Gamma), \\
0, \quad \text { otherwise }
\end{array}\right.
$$

This Markov process is irreducible since for any $e, e^{\prime} \in E \Gamma$ there exists a reduced path in $\Gamma$ with initial edge $e$ and terminal edge $e^{\prime}$. The graph $\Gamma$ has $(m k-m) /(m-2)$ nonoriented edges and $(2 m k-2 m) /(m-2)$ oriented edges. The uniform distribution $\mu_{0}$ on $E \Gamma$, given by $\mu_{0}(e)=\frac{m-2}{2 m k-2 m}$ for every $e \in E \Gamma$, is obviously invariant with respect to our Markov process. Since the process is irreducible, $\mu_{0}$ is the only invariant distribution on $E \Gamma$.

Let $\mu$ be the distribution on $E \Gamma$ which is uniformly distributed on the $m$ oriented edges starting with the base-vertex $p$. In other words, $\mu(e)=1 / m$ if $o(e)=p$ and $\mu(e)=0$ if $o(e) \neq p$.

Let $w_{t}=e_{1}, \ldots, e_{t}$ be a trajectory of our process of length $t$. Let $\epsilon>0$ and $e \in E \Gamma$. By Law of Large Numbers we have

$$
\lim _{t \rightarrow \infty} \mathbb{P}_{\mu}\left(\left|\frac{\left\langle e, w_{t}\right\rangle}{t}-\frac{m-2}{2 m k-2 m}\right|>\epsilon\right)=0 .
$$

Let $\epsilon>0$ be arbitrary. Then there is $t_{0} \geq 1$ such that for every $t \geq t_{0}$ and for every $e \in E \Gamma$ we have

Denote

$$
\mathbb{P}_{\mu}\left(\left|\frac{\left\langle e, w_{t}\right\rangle}{t}-\frac{m-2}{2 m k-2 m}\right| \leq \epsilon\right) \geq \frac{1}{2} .
$$

$$
\begin{gathered}
R(t, \epsilon)=\{w \in \mathcal{P}(\Gamma): w \text { consists of } t \text { edges, and for every } e \in E \Gamma \\
\left.\left|\frac{\langle e, w\rangle}{t}-\frac{m-2}{2 m k-2 m}\right| \leq \epsilon\right\} .
\end{gathered}
$$

Thus for $t \geq t_{0}$

$$
\# R(t, \epsilon) \geq(1 / 2) \cdot m \cdot(m-1)^{t-1} \geq(m-1)^{t-1} .
$$


The volume of $\Gamma$ is equal to one and hence $\sum_{e \in E \Gamma} L_{\mathcal{L}}(e)=2$.

Then for every $w \in R(t, \epsilon)$ we have:

$$
\begin{gathered}
L_{\mathcal{L}}(w)=\sum_{e \in E \Gamma}\langle e, w\rangle L_{\mathcal{L}}(e) \leq \\
\sum_{e \in E \Gamma}\left(\frac{t(m-2)}{2 m k-2 m}+t \epsilon\right) L_{\mathcal{L}}(e)=\left(\frac{t(m-2)}{2 m k-2 m}+t \epsilon\right) \sum_{e \in E \Gamma} L_{\mathcal{L}}(e)= \\
\left(\frac{t(m-2)}{2 m k-2 m}+t \epsilon\right) \cdot 2=\frac{t(m-2)}{m k-m}+2 t \epsilon .
\end{gathered}
$$

Let $c=\min _{e \in E \Gamma} e^{s L(e)}$. Then Lemma 8.2 together with $(\nabla)$ and $(\boldsymbol{\omega})$ imply that for every integer $t \geq 1$

$$
\sum_{i j}\left(A_{\mathcal{L}}(s)^{t}\right)_{i j} \geq c(m-1)^{t-1} e^{-s \frac{t(m-2)}{m k-m}+2 t \epsilon}=\frac{c}{m-1}\left[(m-1) e^{-s \frac{(m-2)}{m k-m}+2 \epsilon}\right]^{t}
$$

For $s=h(\mathcal{L})$ the matrix $A_{\mathcal{L}}(s)$ has spectral radius 1 and therefore

$$
(m-1) e^{-s \frac{(m-2)}{m k-m}+2 \epsilon} \leq 1
$$

and hence

$$
s \geq \frac{m k-2}{m-2}(\log (m-1)+2 \epsilon)
$$

Since this is true for all $\epsilon>0$, this implies that

$$
s \geq \frac{m k-m}{m-2} \log (m-1)
$$

The following easy computation shows that the bound in Proposition 8.1 is realized by the "uniform" volume-one metric structure, where all edges have equal lengths.

Lemma 8.3. Let $\Gamma$ be as in Proposition 8.1. Let $\mathcal{L}_{0}$ be the "uniform" volume-one metric structure on $\Gamma$, that is $L_{\mathcal{L}_{0}}(e)=\frac{m-2}{m k-m}$ for every $e \in E \Gamma$. Then

$$
h\left(\mathcal{L}_{0}\right)=\frac{m(k-1)}{m-2} \log (m-1)
$$

Proof. A direct check shows that for $s_{0}=\frac{m(k-1)}{m-2} \log (m-1)$ we have

$$
A_{\mathcal{L}_{0}}\left(s_{0}\right)\left[\begin{array}{c}
1 \\
1 \\
\vdots \\
1
\end{array}\right]=\left[\begin{array}{c}
1 \\
1 \\
\vdots \\
1
\end{array}\right] .
$$

Therefore the Perron-Frobenius eigenvalue of $A_{\mathcal{L}_{0}}\left(s_{0}\right)$ is equal to 1 and hence $h\left(\mathcal{L}_{0}\right)=s_{0}$, as claimed.

Note that for the case $m=3$, corresponding to regular trivalent graphs, we have $\frac{m k-m}{m-2} \log (m-1)=(3 k-3) \log 2$. We are now ready to prove part $(1)$ of Theorem $\mathrm{B}$ from the Introduction. 
Theorem 8.4. Let $F_{k}$ be a free group of finite rank $k \geq 2$, and let $\mathcal{M}_{k}:=$ $C V\left(F_{k}\right) / \operatorname{Out}\left(F_{k}\right)$ be the moduli space. For the function $\bar{h}: \mathcal{M}_{k} \rightarrow \mathbb{R}$ we have

$$
\min \bar{h}=(3 k-3) \log 2 .
$$

This minimum is realized by any regular trivalent connected graph $\Gamma$ with $\pi_{1}(\Gamma) \cong$ $F_{k}$ (so that $\Gamma$ has $3 k-3$ non-oriented edges), where each edge of $\Gamma$ is given length $1 /(3 k-3)$.

Proof. The moduli space $\mathcal{M}_{k}$ is a union of finitely many open simplices, corresponding to taking all volume-one metric structures on all the possible minimal graphs with fundamental group free of rank $k$.

Let $(\Gamma, \mathcal{L}) \in \mathcal{M}_{k}$. Then $(\Gamma, \mathcal{L})$ can be approximated in $\mathcal{M}_{k}$ by trivalent metric graphs. By Proposition 8.1 for all of these trivalent graphs the volume entropy is $\geq(3 k-3) \log 2$. Since $\bar{h}$ is continuous on $\mathcal{M}_{k}$, it follows that $\bar{h}(\mathcal{L}) \geq(3 k-3) \log 2$ as well. Together with Lemma 8.3 this implies the conclusion of Theorem 8.4

The following is part (3) of Theorem B from the Introduction.

Theorem 8.5. Let $F_{k}$ be a free group of finite rank $k \geq 2$, and let $\mathcal{M}_{k}:=$ $C V\left(F_{k}\right) /$ Out $\left(F_{k}\right)$ be the moduli space. Then

$$
\sup _{\mathcal{M}_{k}} \bar{h}=\infty .
$$

Proof. First note that it suffices to prove the statement of the theorem for $k=2$. Indeed, suppose we know that $\sup _{\mathcal{M}_{2}} \bar{h}=\infty$ and let $k>2$ be arbitrary.

Let $(\Gamma, \mathcal{L})$ be a finite volume-one connected metric graph with $\pi_{1}(\Gamma) \cong F_{2}$. Let $X=\widetilde{(\Gamma, p)}$, where $p$ is a vertex of $\Gamma$, and let $X$ be endowed with a metric $d_{\mathcal{L}}$ induced by $\mathcal{L}$. Denote by $d$ the corresponding metric on $\partial X$.

Put $\Gamma_{1}$ to be the wedge at $p$ of the graph $\Gamma$ with $k-2$ loop-edges. Let $\mathcal{L}_{1}$ be the metric structure on $\Gamma_{1}$ where each of the new loop-edges is given length $\frac{1}{2(k-2)}$ and where $\mathcal{L}_{1}$ restricted to $\Gamma$ is $\mathcal{L} / 2$. Then $\mathcal{L}_{1}$ has volume one and $\pi_{1}\left(\Gamma_{1}, p\right) \cong F_{k}$.

Let $X_{1}=\widetilde{\left(\Gamma_{1}, p\right)}$, endowed with the induced metric $d_{\mathcal{L}_{1}}$. Denote by $d_{1}$ the corresponding metric on $\partial X_{1}$. Then $X_{1}$ contains an isometrically embedded copy of $\left(X, \frac{d_{\mathcal{C}}}{2}\right)$ and hence $\left(\partial X_{1}, d_{1}\right)$ contains an isometrically embedded copy of $\left(\partial X, d^{1 / 2}\right)$. Taking the square root of a metric doubles the Hausdorff dimension and therefore

$$
h\left(X_{1}\right) \geq 2 h(X) .
$$

In particular, $h(X) \rightarrow \infty$ implies $h\left(X_{1}\right) \rightarrow \infty$.

Thus we may assume that $k=2$. Let $\Gamma$ be the wedge of two loop-edges at a single vertex. Denote $E \Gamma=\{g, \bar{g}, f, \bar{f}\}$. Let $\mathcal{L}$ be a volume-one metric structure on $\Gamma$ and denote $x=L(g), y=L(f)$, so that $x+y=1$ and $x, y>0$. Then $\bar{h}(\mathcal{L})$ is the unique number $s>0$ such that $\Phi_{\mathcal{L}}(s)=1$. The condition $\Phi_{\mathcal{L}}(s)=1$ is equivalent to the existence of a positive vector $Y>0$ such that $A_{\mathcal{L}}(s) Y=Y$.

The symmetry considerations imply that $Y_{g}=Y_{\bar{g}}$ and $Y_{f}=Y_{\bar{f}}$. Denote $a=$ $Y_{g}=Y_{\bar{g}}$ and $b=Y_{f}=Y_{\bar{f}}$. Then the system $A_{\mathcal{L}}(s) Y=Y$ is:

$$
\left\{\begin{array}{l}
e^{-s x}(a+2 b)=a, \\
e^{-s y}(b+2 a)=b,
\end{array}\right.
$$


Up to re-scaling we may assume $b=1$, so that the above system transforms into the equation

$$
4=\left(e^{s x}-1\right)\left(e^{s y}-1\right) .
$$

Since the volume is equal to one we have $y=1-x$. For $0<x<1$ denote by $s(x)$ the unique value $s>0$ such that the equation (\#) holds.

We claim that $s(x) \rightarrow \infty$ as $x \rightarrow 0+$. Indeed, suppose not. Then there exists a sequence $x_{n}>0$, with $\lim _{n \rightarrow \infty} x_{n}=0$ such that for the corresponding values $s_{n}=$ $s\left(x_{n}\right)$ we have $s_{n} \leq M$, where $0<M<\infty$. Also, denote $y_{n}=1-x_{n}$. Then $e^{s_{n} y_{n}}-1 \leq e^{M}-1=: K$. Since $0<s_{n} \leq M$ and $\lim _{n \rightarrow \infty} x_{n}=0$, we have $\lim _{n \rightarrow \infty} e^{s_{n} x_{n}}-1=0$. Therefore there exists $m>1$ such that $0<e^{s_{m} x_{m}}-1<1 / K$. Together with $0<e^{s_{m} y_{m}}-1 \leq K$ this implies

$$
\left(e^{s_{m} x_{m}}-1\right)\left(e^{s_{m} y_{m}}-1\right) \leq K \cdot(1 / K)=1<4,
$$

yielding a contradiction.

\section{UNIQUENESS OF CRITICAL POINTS FOR VOLUME ENTROPY}

In this Section we will compute the derivative of the volume entropy function $h$ (see also 34 for another proof) and, as a consequence, prove Part (2) of Theorem $[$ For the remainder of this section, unless specified otherwise let $\Gamma$ be a finite connected graph without degree-one vertices and with the fundamental group free of rank $k \geq 2$. Let $n$ be the number of oriented edges of $\Gamma$ and let $N=n / 2$ be the number of non-oriented edges of $\Gamma$. Let $Q$ be the set of all volume-one positive quasi-metric structures on $\Gamma$. We identify $Q$ with the open simplex of dimension $n-1$ in $\mathbb{R}^{n}$ :

$$
Q=\left\{\left(L_{1}, \ldots, L_{n}\right) \in \mathbb{R}^{n}: \sum_{i=1}^{n} L_{i}=2 \text {, and } L_{i}>0 \text { for } i=1, \ldots, n\right\} .
$$

We will be using the notations from Section 4 The proof of Proposition 4.6 shows that $h$ extends to a smooth function $s: V \rightarrow \mathbb{R}$ where $V$ is an open neighborhood of $Q$ in $\mathbb{R}^{n}$. Recall the notation $s=s(\bar{L})$ from Corollary 4.7

We are now going to compute the partial derivatives $\frac{\partial s}{\partial L_{i}}$. For that we will use the proof of Proposition 4.6] and the following statement from Seneta's book [37]:

Proposition 9.1 (see Theorem 1.5 in 37). Let $A \geq 0$ be a nonzero irreducible $n \times n$ matrix. Then

$$
\operatorname{adj}\left(r(A) I_{n}-r(A)\right)=\epsilon Y Z,
$$

where $Z$ and $Y$ are left and right Perron-Frobenius eigenvectors of $A$ accordingly and where $\epsilon \in\{-1,1\}$.

Proposition 9.2. Let $\mathcal{L}=\left(L_{1}, \ldots, L_{n}\right) \in Q$. Then, at the point $\mathcal{L}$, for $i=1, \ldots, n$ we have

$$
\frac{\partial s}{\partial L_{i}}=s b_{i} e^{-s L_{i}}=2 \epsilon s z_{i}\|Y\|^{2} e^{-s L_{i}} \operatorname{det}(J)^{-1},
$$

where $Z=\left[z_{1}, \ldots, z_{n}\right]$ is a right Perron-Frobenius eigenvector of $A=A_{\mathcal{L}}(s)$; where $J$ and $Y$ are as in the proof of Proposition $\left[4.6\right.$; where $\left[b_{1} \ldots b_{n} b_{n+1}\right]$ is the last row of $J^{-1}$ and where $\epsilon \in\{-1,1\}$. 
Proof. Recall that in the proof of Proposition 4.6 the function $s: V \rightarrow \mathbb{R}$ is defined via the Implicit Function Theorem applied to the system of equations (!). By the Implicit Function Theorem via differentiating (!) we also have at $\mathcal{L} \in Q$ :

$$
\begin{array}{r}
\frac{\partial\left(y_{1}, \ldots, y_{n}, s\right)}{\partial\left(L_{1}, \ldots, L_{n}\right)}=-\left[\frac{\partial\left(F_{1}, \ldots, F_{n}, F_{n+1}\right)}{\partial\left(y_{1}, \ldots, y_{n}, s\right)}\right]^{-1} \frac{\partial\left(F_{1}, \ldots, F_{n}, F_{n+1}\right)}{\partial\left(L_{1}, \ldots, L_{n}\right)}= \\
=-J^{-1}\left[\begin{array}{ccccc}
-s e^{-s L_{1}} & 0 & 0 & \ldots & 0 \\
0 & -s e^{-s L_{2}} & 0 & \ldots & 0 \\
\vdots & \vdots & \vdots & \ldots & \vdots \\
0 & 0 & 0 & \ldots & -s e^{-s L_{n}} \\
0 & 0 & 0 & \ldots & 0
\end{array}\right]
\end{array}
$$

where

$$
J=\left[\begin{array}{cccccc}
a_{11}-1 & a_{12} & a_{13} & \ldots & a_{1 n} & -L_{1} y_{1} \\
a_{21} & a_{22}-1 & a_{23} & \ldots & a_{2 n} & -L_{2} y_{2} \\
\vdots & \vdots & \vdots & \ldots & \vdots & \vdots \\
a_{i 1} & a_{i 2} & a_{i 3} & \ldots & a_{i n} & -L_{i} y_{i} \\
\vdots & \vdots & \vdots & \ldots & \vdots & \vdots \\
a_{n 1} & a_{n 2} & a_{n 3} & \ldots & a_{n n}-1 & -L_{n} y_{n} \\
2 y_{1} & 2 y_{2} & 2 y_{3} & \ldots & 2 y_{n} & 0
\end{array}\right]
$$

Thus to compute $\frac{\partial s}{\partial\left(L_{1}, \ldots, L_{n}\right)}$ we need to know the last row $\left[b_{1} \ldots b_{n} b_{n+1}\right]$ of $J^{-1}$. Then

$$
\frac{\partial s}{\partial L_{i}}=s b_{i} e^{-s L_{i}}
$$

for $i=1, \ldots, n$. Note that the value $b_{n+1}$ is actually not needed since the last row of $\frac{\partial\left(F_{1}, \ldots, F_{n}, F_{n+1}\right)}{\partial\left(L_{1}, \ldots, L_{n}\right)}$ consists entirely of zeros.

Since $J^{-1}=\operatorname{det}(J)^{-1} \operatorname{adj}(J)$, we have:

$$
b_{i}=-\operatorname{det}(J)^{-1} \operatorname{det}\left[\begin{array}{ccccc}
a_{11}-1 & a_{12} & a_{13} & \ldots & a_{1 n} \\
a_{21} & a_{22}-1 & a_{23} & \ldots & a_{2 n} \\
\vdots & \vdots & \vdots & \ldots & \vdots \\
2 y_{1} & 2 y_{2} & 2 y_{3} & \ldots & 2 y_{n} \\
\vdots & \vdots & \vdots & \ldots & \vdots \\
a_{n 1} & a_{n 2} & a_{n 3} & \ldots & a_{n n}-1
\end{array}\right]
$$

where the $\left\{y_{j}\right\}$ occur in the $i$-th row. Recall that $Y=\left[y_{1}, y_{2} \ldots, y_{n}\right]^{T}$ is a right Perron-Frobenius eigenvector of $A=A_{\mathcal{L}}(s)$. By Proposition 9.1 we have $\operatorname{adj}(A-$ $\left.I_{n}\right)=-\epsilon Y Z$ where $Z=\left[z_{1}, \ldots, z_{n}\right]$ is a left Perron-Frobenius eigenvector of $A$. Denote $R=\operatorname{adj}\left(A-I_{n}\right)$. Then, by taking the $i$-th row decomposition of the determinant in (!!) we see that $b_{i}$ is equal to the scalar product of $-\operatorname{det}(J)^{-1}\left[2 y_{1}, \ldots, 2 y_{n}\right]$ and the $i$-th column of $R$. Since $R=-\epsilon Y Z$, the $i$-th column of $R$ is $-\epsilon z_{i} Y$. Hence $b_{i}=2 \epsilon z_{i}\|Y\|^{2} \operatorname{det}(J)^{-1}$.

Then by $(+)$ we have $\frac{\partial s}{\partial L_{i}}=s b_{i} e^{-s L_{i}}=2 \epsilon s z_{i}\|Y\|^{2} e^{-s L_{i}} \operatorname{det}(J)^{-1}$, as required.

Proposition 9.3. Suppose that $\Gamma$ is m-regular for some $m \geq 3$. Let $\mathcal{L} \in Q$ be a critical point of $\left.s\right|_{Q}$. Then $L_{1}=L_{2}=\cdots=L_{n}$. 
Proof. By the Lagrange multipliers method at a critical point of $\left.s\right|_{Q}$ we have that the gradient of $s$ is parallel to the vector $(1,1, \ldots, 1)$, that is, at such a point

$$
\frac{\partial s}{\partial L_{1}}=\frac{\partial s}{\partial L_{2}}=\cdots=\frac{\partial s}{\partial L_{n}} \text {. }
$$

By the earlier computations we have $\frac{\partial s}{\partial L_{i}}=s b_{i} e^{-s L_{i}}=2 \epsilon s z_{i}\|Y\|^{2} e^{-s L_{i}} \operatorname{det}(J)^{-1}$. Then by (!!!) at a critical point $\mathcal{L}$ of $\left.s\right|_{Q}$ we have

$$
z_{1} e^{-s L_{1}}=z_{2} e^{-s L_{2}}=\cdots=z_{n} e^{-s L_{n}},
$$

where $Z=\left[z_{1}, z_{2}, \ldots, z_{n}\right]$ is a left Perron-Frobenius eigenvector of $A$. Up to rescaling we can choose $Z$ so that the first coordinate of $Z$ is $e^{s L_{1}}$. Then by the above equation $Z=\left[e^{s L_{1}}, e^{s L_{2}}, \ldots, e^{s L_{n}}\right]$.

Now we use the fact that $Z A=Z \operatorname{diag}\left(e^{-s L_{i}}\right) M=Z$ which translates into $[1,1, \ldots, 1] M=Z$. Since $\Gamma$ is $m$-regular, in every column of $M$ there are exactly $m-1$ nonzero entries, each equal to 1 . Then $[1,1, \ldots, 1] M=Z$ translates into $z_{1}=\cdots=z_{n}=m-1$. Since we have chosen $Z$ so that $z_{i}=e^{s L_{i}}$, this implies $L_{1}=L_{2}=\cdots=L_{n}$, as required.

The following statement is a corollary of a result obtained by Robert 35] and Rivin 34.

Proposition 9.4. Let $\Gamma$ be a finite connected graph without degree-one vertices with $\pi_{1}(\Gamma)$ free of rank $\geq 2$ and with $N$ non-oriented edges.

Let $U=U(\Gamma)$ be the set of all metric graph structures on $\Gamma$. Thus $U$ is identified with

$$
U=\left\{\left(x_{1}, \ldots, x_{N}\right) \in \mathbb{R}^{N}: x_{i}>0, i=1, \ldots, N\right\} .
$$

Then the volume entropy $h: U \rightarrow \mathbb{R}$ is a convex function on $U$.

The following is part (2) of Theorem $\mathbb{B}$ from the Introduction.

Theorem 9.5. Let $\Gamma$ be a finite connected graph without degree-one and degreetwo vertices and with fundamental group free of rank $k \geq 1$. Let $\mathcal{L}$ be a volume-one metric structure such that $h(\mathcal{L})=(3 k-3) \log 2$. Then $\Gamma$ is a regular trivalent graph and all edges of $\Gamma$ have equal lengths in $\mathcal{L}$.

Proof. Case 1. Suppose first that $\Gamma$ is trivalent. Then Proposition 8.1 implies that $\mathcal{L}$ is a point of minimum of $\left.s\right|_{Q}$. Therefore $\mathcal{L}$ is a critical point of $\left.s\right|_{Q}$. Hence by Proposition 9.3 all edges have equal lengths in $\mathcal{L}$.

Case 2. Suppose now that $\Gamma$ is not trivalent. Then there exists a trivalent graph $\Gamma^{\prime}$ and a sequence of volume-one metric structures $\mathcal{L}_{t}^{\prime}$ on $\Gamma^{\prime}$ such that $\left(\Gamma^{\prime}, \mathcal{L}_{t}^{\prime}\right)$ converges to $(\Gamma, \mathcal{L})$ in the moduli space $C V\left(F_{k}\right) / \operatorname{Out}\left(F_{k}\right)$ as $t \rightarrow \infty$. Moreover, since $\Gamma$ is not trivalent, there is an edge $e$ of $\Gamma^{\prime}$ whose $\mathcal{L}_{t^{-}}$-length converges to 0 as $t \rightarrow \infty$, so that, after possibly passing to a subsequence, the points $\mathcal{L}_{t}^{\prime}$ converge to a point in the boundary of the open simplex $U\left(\Gamma^{\prime}\right)$.

By continuity of $h$ we have $\lim _{t \rightarrow \infty} h\left(\mathcal{L}_{t}^{\prime}\right)=(3 k-3) \log 2$. Let $\mathcal{L}_{0}$ be the volume-one metric structure on $\Gamma^{\prime}$ where all edges have equal length. Let $S$ be the set of all points in $U=U(\Gamma)$ at distance $\epsilon>0$ where $\epsilon$ is smaller than the distance from $\mathcal{L}_{0}$ to the boundary of $U$. For sufficiently large $t$ the segments $\left[\mathcal{L}_{0}, \mathcal{L}_{t}^{\prime}\right]$ intersect $S$ in one point denoted $\mathcal{L}_{t}^{\prime \prime}$. By convexity of $h$ we have $h\left(\mathcal{L}_{t}^{\prime \prime}\right) \leq \max \left\{h\left(\mathcal{L}_{0}\right), h\left(\mathcal{L}_{t}^{\prime}\right)\right\}$. Since $S$ is compact, after passing to a subsequence we have $\lim _{t \rightarrow \infty} \mathcal{L}_{t}^{\prime \prime}=\mathcal{L}^{\prime \prime} \in S$. Since $\lim _{t \rightarrow \infty} h\left(\mathcal{L}_{t}^{\prime}\right)=h\left(\mathcal{L}_{0}\right)=(3 k-3) \log 2$, it follows that $h\left(\mathcal{L}^{\prime \prime}\right) \leq(3 k-3) \log 2$ and 
therefore $h\left(\mathcal{L}^{\prime \prime}\right)=(3 k-3) \log 2$ by Theorem 8.4. By Case 1 all edges of $\mathcal{L}^{\prime \prime}$ have equal length, which contradicts the fact that $\mathcal{L}^{\prime \prime} \neq \mathcal{L}_{0}$.

\section{REFERENCES}

[1] G. Besson, G. Courtois, and S. Gallot, Entropies et rigidités des espaces localement symétriques de courbure strictement négative. Geom. Funct. Anal. 5 (1995), no. 5, 731-799

[2] G. Besson, G. Courtois, and S. Gallot, Minimal entropy and Mostow's rigidity theorems. Ergodic Theory Dynam. Systems 16 (1996), no. 4, 623-649

[3] F. Bonahon, Bouts des variétés hyperboliques de dimension 3. Ann. of Math. (2) 124 (1986), no. 1, 71-158

[4] F. Bonahon, The geometry of Teichmüller space via geodesic currents. Invent. Math. 92 (1988), no. 1, 139-162

[5] M. Bourdon, Structure conforme au bord et flot géodésique d'un CAT(-1)-espace, Thèse, Université de Paris-Sud, 1993.

[6] M. Bridgeman, Average bending of convex pleated planes in hyperbolic three-space. Invent. Math. 132 (1998), no. 2, 381-391

[7] M. Bridgeman, and E. Taylor, Length distortion and the Hausdorff dimension of limit sets. Amer. J. Math. 122 (2000), no. 3, 465-482

[8] M. Burger, and S. Mozes, CAT(-1)-spaces, divergence groups and their commensurators. J. Amer. Math. Soc. 9 (1996), no. 1, 57-93

[9] M. Coornaert, Mesures de Patterson-Sullivan sur le bord d'un espace hyperbolique au sens de Gromov. Pacific J. Math. 159 (1993), no. 2, 241-270

[10] M. Coornaert, and A. Papadopoulos, Upper and lower bounds for the mass of the geodesic flow on graphs. Math. Proc. Cambridge Philos. Soc. 121 (1997), no. 3, 479-493

[11] M. Coornaert, and A. Papadopoulos, Spherical functions and conformal densities on spherically symmetric CAT(-1)-spaces Trans. Amer. Math. Soc. 351 (1999), no. 7, 27452762

[12] M. Culler, K. Vogtmann, Moduli of graphs and automorphisms of free groups. Invent. Math. 84 (1986), no. 1, 91-119.

[13] G. Edgar, Measure, topology, and fractal geometry. Springer-Verlag, New York, 1990

[14] A. Furman, Coarse-geometric perspective on negatively curved manifolds and groups, in "Rigidity in Dynamics and Geometry (editors M. Burger and A. Iozzi)", Springer 2001, 149-166

[15] E. Ghys and P. de la Harpe (editors), Sur les groupes hyperboliques d'après Mikhael Gromov, Birkhäuser, Progress in Mathematics series, vol. 83, 1990

[16] L. Guillopé, Entropies et spectres. Osaka J. Math. 31 (1994), no. 2, 247-289.

[17] U. Hamenstädt, Ergodic properties of function groups. Geom. Dedicata 93 (2002), 163-176

[18] S. Hersonsky, and F. Paulin, On the rigidity of discrete isometry groups of negatively curved spaces. Comment. Math. Helv. 72 (1997), no. 3, 349-388

[19] V. Kaimanovich, Bowen-Margulis and Patterson measures on negatively curved compact manifolds. Dynamical systems and related topics (Nagoya, 1990), 223-232, Adv. Ser. Dynam. Systems, 9, World Sci. Publishing, River Edge, NJ, 1991

[20] V. Kaimanovich, I. Kapovich and P. Schupp, The Subadditive Ergodic Theorem and generic stretching factors for free group automorphisms, Israel J. Math, to appear; http://arxiv.org/abs/math.GR/0504105

[21] I. Kapovich, The frequency space of a free group, Internat. J. Alg. Comput. 15 (2005), no. 5-6, 939-969

[22] I. Kapovich, Currents on free groups, AMS Contemporary Mathematics Series, to appear; http://arxiv.org/abs/math.GR/0412128

[23] I. Kapovich, G. Levitt, P. Schupp and V. Shpilrain, Translation equivalence in free groups, Transact. Amer. Math. Soc., to appear; http://www.arxiv.org/math.GR/0409284

[24] A. Katok, Entropy and closed geodesics. Ergodic Theory Dynam. Systems 2 (1982), no. 3-4, 339-365

[25] S. Lim, Minimal Volume Entropy on Graphs, http://arxiv.org/abs/math.GR/0506216

[26] R. Lyons, Equivalence of boundary measures on covering trees of finite graphs, Ergodic Theory Dynam. Systems 14 (1994), no. 3, 575-597 
[27] R. Martin, Non-Uniquely Ergodic Foliations of Thin Type, Measured Currents and Automorphisms of Free Groups, PhD Thesis, UCLA, 1995

[28] R. D. Mauldin and S. Williams, Hausdorff dimension in graph directed constructions. Trans. Amer. Math. Soc. 309 (1988), no. 2, 811-829

[29] T. Nagnibeda, Random walks on trees and Ramanujan graphs. in: "Random walks and Geometry", ed. V. Kaimanovich, de Gruyter, 2004, pp. 487-500

[30] T. Nagnibeda, W. Woess, Random walks on trees with finitely many cone types. J. Theoret. Probab. 15 (2002), pp. 383-422

[31] S. J. Patterson, The limit set of a Fuchsian group. Acta Math. 136 (1976), pp. 241-273

[32] F. Paulin, On the critical exponent of a discrete group of hyperbolic isometries. Differential Geom. Appl. 7 (1997), no. 3, 231-236

[33] J.-C. Picaud, Cohomologie bornée des surfaces et courants géodésiques. Bull. Soc. Math. France 125 (1997), no. 1, 115-142

[34] I. Rivin, Growth in free groups (and other stories), preprint, 1999, http://www.arxiv.org/math.CO/9911076

[35] G. Robert Entropie et graphes. Prépublication 182, ENS Lyon, 1996

[36] D. Sarić, Geodesic currents and Teichmüller space, Topology 44 (2005), no. 1, 99-130

[37] E. Seneta, Nonnegative matrices and Markov chains. Second edition. Springer Series in Statistics. Springer-Verlag, New York, 1981

[38] D. Sullivan, The density at infinity of a discrete group of hyperbolic motions. Inst. Hautes Études Sci. Publ. Math. 50 (1979), 171-202

[39] K. Vogtmann, Automorphisms of Free Groups and Outer Space, Geometriae Dedicata 94 (2002), 1-31

Department of Mathematics, University of Illinois at Urbana-Champaign, 1409 West Green Street, Urbana, IL 61801, USA

http://www.math.uiuc.edu/ Kapovich/

E-mail address: kapovich@math.uiuc.edu

Section de mathématiques, Université de Genève, 2-4, rue du Lièvre, c.p. 64, 1211 Genève, Switzerland

http://www.unige.ch/math/folks/nagnibeda

E-mail address: tatiana.smirnova-nagnibeda@math.unige.ch 